\title{
Optimal feedback control strategies for periodic delayed systems
}

\author{
Morad Nazari · Eric A. Butcher • Oleg A. Bobrenkov
}

Received: 17 July 2013 / Revised: 16 December 2013 / Accepted: 24 December 2013 / Published online: 17 January 2014

(c) Springer-Verlag Berlin Heidelberg 2014

\begin{abstract}
In this study, three strategies based on infinitedimensional Floquet theory, Chebyshev spectral collocation, and the Lyapunov-Floquet transformation (LFT) are proposed for optimal feedback control of linear time periodic delay differential equations using periodic control gains. First, a periodic-gain discrete-delayed feedback control is implemented where optimization of the control gains is included to obtain the minimum spectral radius of the closedloop response. Second, a large set of ODEs is obtained using the Chebyshev spectral continuous time approximation, after which optimal (time-varying LQR) control is used to obtain a periodic-gain distributed-delayed feedback control. The third strategy involves the use of both CSCTA and the reduced LFT, along with either pole-placement or time-invariant LQR used on a linear time invariant auxiliary system, to obtain a periodic-gain non-delayed feedback control that asymptotically stabilizes the original system. The delayed Mathieu equation is used as an illustrative example for all three control strategies.
\end{abstract}

Keywords Periodic delay differential equations - Floquet theory $\cdot$ Chebyshev spectral collocation $\cdot$ Lyapunov-Floquet transformation $\cdot$ Optimal feedback control

M. Nazari $(\bowtie)$. E. A. Butcher

Department of Aerospace and Mechanical Engineering, University

of Arizona, Tucson, AZ 85721, USA

e-mail: mozari82@yahoo.com

O. A. Bobrenkov

KABA Lodging Systems Russia, 43 Izmaylovsky blvd,

Moscow 105264, Russia

\section{Introduction}

Many strategies have been utilized to control time-delayed systems or to design delayed feedback control laws for nondelayed systems in the case that the current states are unavailable for feedback. For linear time-invariant (LTI) control systems with delay, the stabilization problem is to design a control law such that all of the closed-loop poles (eigenvalues) lie in the left-half plane. If pole placement is desired to ensure a specified response, however, one is faced with the problem that infinitely many poles should be specified, while the number of control gains is finite. One strategy is to assign the place of the dominant poles only or to specify only the spectral abscissa [1]. An alternative strategy is to use an infinite (or large) number of control gains to control all (or many) of the poles via the use of feedback control with either distributed delay and/or periodic control gains with an arbitrary number of Fourier coefficients [2]. The use of time-periodic control gains results in the closed-loop system having the form of time-periodic delay differential equations (DDEs), for which stability analysis requires the use of the infinite-dimensional Floquet theory [3].

Another strategy for delayed feedback control is that of finite spectrum assignment, in which the resulting closedloop system has only a finite number of poles that can be assigned arbitrarily and thus requires only a finite number of control gains to be specified. Two techniques to implement this strategy include the use of a feedback based on a prediction of the state with distributed delay, e.g. [4-7], and also utilizing a special case of periodic controllers called actand-wait control where the feedback term is switched on and off periodically in time [8-10]. The advantage in this control strategy is that if the switch-off (waiting) period is longer than the feedback delay, then the system can be transformed 
to a discrete map of finite dimension thus presenting a finite spectrum assignment problem.

Recently, new computational tools have been demonstrated that enable many of the traditional tools for timeinvariant non-delayed systems to be successfully applied to DDEs. These include the Chebyshev spectral continuous time approximation (CSCTA) and reduced LyapunovFloquet transformation (RLFT). Other methods that convert a system of DDEs to a non-delayed system of ODEs are finite differences, which was employed for feedback control of delayed systems in [11], and the Galerkin-projection [12] technique. By using the CSCTA technique [13], a periodic DDE is transformed into a system of periodic ODEs using Chebyshev spectral collocation where the dimension of the resulting system depends on the number of collocation points used. In contrast, the Chebyshev collocation method used in [14] (together with semidiscretization $[2,15]$ or temporal finite element analysis [16]) transforms the original DDE into a large-dimensional discrete map instead of a system of ODEs.

By application of the LFT, the analysis of linear timeperiodic ODEs can be confined to that of an equivalent set of constant ODEs [17]. This approach was first successfully implemented for control of linear time-periodic systems in $[18,19]$ and chaos and bifurcation control [20,21]. For periodic time-delayed systems discretized using CSCTA, however, due to the inaccurate eigenvalues of the monodromy matrix (Floquet multipliers) clustered around the origin, it is impractical to use the full LFT matrix [22]. Therefore, in that study only dominant Floquet multipliers were considered to obtain the reduced LFT (RLFT) matrix, and the stability and time-response of a linear time periodic DDE was investigated by transforming it into a low-dimensional constant ODE.

In this study, three optimal feedback control strategies are proposed for linear periodic time delayed systems using current or delayed state feedback with periodic control gains. In the first strategy, which extends the constant-gain delayed feedback control strategy in $[2,11,15]$, delayed state feedback control with discrete delay is implemented and the stability of the closed-loop response is investigated in the parameter space of available control gains using Floquet theory, including the optimization of the control gains for minimum spectral radius of the closed-loop response. In the second strategy, optimal (time-varying LQR) delayedfeedback control with distributed delay is used with the CSCTA-approximated system by directly solving the periodic Riccati equation (PRE) backwards in time or by solving the corresponding algebraic Riccati equation (ARE) from which the periodic control gains are obtained. The third strategy involves use of both CSCTA and RLFT as well as poleplacement or time-invariant LQR design for auxiliary LTI systems to obtain the control gains that asymptotically stabilize the LTI system, and hence the original system as well, resulting in an optimal non-delayed state feedback control law. The non-delayed counterpart of this strategy is adopted from $[18,19]$. These strategies are illustrated via current or delayed feedback control design of the delayed Mathieu equation. The control efforts and closed-loop responses are compared for these strategies. In each strategy, the use of more Fourier terms in the periodic control gains generally leads to better optimization of the cost function or closedloop spectral radius. This study is unique in that the combined use of CSCTA and RLFT has not been investigated in control design for delayed periodic systems, while optimal feedback control of such systems has received very little attention.

\section{Problem statement and CSCTA}

Consider the $q$-dimensional linear system with a single point delay given by

$$
\dot{\mathbf{x}}=\mathbf{A}_{1}(t) \mathbf{x}(t)+\mathbf{A}_{2}(t) \mathbf{x}(t-\tau)+\mathbf{B}(t) \mathbf{u}(t)
$$

$\mathbf{x}(t)=\phi(t), \quad-\tau \leq t \leq 0$

in which $\mathbf{x}(t) \in \mathbb{R}^{q}, \mathbf{u}(t) \in \mathbb{R}^{p}$, and the matrices $\mathbf{A}_{1}(t), \mathbf{A}_{2}(t)$, and $\mathbf{B}(t)$ are periodic in $t$ with period $T$. In general, the abstract representation of Eq. (1) is the evolution of the initial function $\phi$ in a Banach space, i.e. $\dot{\mathcal{Y}}(t)=\mathcal{A}(t) \mathcal{Y}(t)+\mathcal{B}(t) \mathbf{u}(t)$, with $\mathcal{Y}(0)=\phi$. In the case of the uncontrolled system with constant $\mathbf{A}_{1}$ and $\mathbf{A}_{2}$, the timeinvariant operator $\mathcal{A}$ is the infinitesimal generator of the solution operator $\mathcal{T}(t): \mathcal{C}\left([-\tau, 0], \mathbb{R}^{q}\right) \rightarrow \mathcal{C}\left([-\tau, 0], \mathbb{R}^{q}\right)=$ $\frac{\partial \mathcal{Y}(t)}{\partial \phi}$, i.e.

$\mathcal{A} \phi=\frac{d \phi}{d \theta}=\lim _{t \rightarrow 0} \frac{1}{t}(\mathcal{T}(t) \phi-\phi), \quad-\tau \leq \theta<0$

$\mathcal{A} \phi=\mathbf{A}_{1} \mathbf{x}(0)+\mathbf{A}_{2} \mathbf{x}(-\tau), \quad \theta=0$.

For periodic DDEs, however, the operator $\mathcal{A}(t)$ is also periodic [13]. Approximation of the abstract ODE representation with a large-dimensional system of ODEs is the main idea of CSCTA.

In the CSCTA method, the interval $[x(t-\tau), x(t)]$ is discretized into $N=m-1$ subintervals whose unequal lengths are determined from the $m$ Chebyshev collocation points defined on $[-1,1]$. Chebyshev collocation points can be introduced as the projections of the equispaced points on the upper half of the unit circle onto the horizontal axis as

$t_{\alpha}=\cos \frac{\alpha \pi}{N}, \quad \alpha=0,1,2, \ldots, N$.

A Chebyshev spectral differentiation matrix $\mathbf{D}$ is defined as

$$
\begin{aligned}
& D_{11}=\frac{2 N^{2}+1}{6}=-D_{N+1, N+1}, \\
& D_{\beta \beta}=-\frac{t_{\beta}}{2\left(1-t_{\beta}^{2}\right)}, \quad \beta=2, \ldots, N
\end{aligned}
$$


$D_{\alpha \beta}=\frac{c_{\alpha}(-1)^{\alpha+1}}{c_{\beta}\left(t_{\alpha}-t_{\beta}\right)}, \quad \alpha \neq \beta, \quad \alpha, \beta=1,2, \ldots, N+1$,

$c_{\alpha}= \begin{cases}2, & \alpha=1, N+1 \\ 1, & \text { otherwise }\end{cases}$

Application of CSCTA discretizes the infinite-dimensional abstract representation of the DDE system into a $m q$ dimensional set of periodic ODEs, i.e.

$\dot{\mathbf{y}}(t)=\hat{\mathbf{A}}(t) \mathbf{y}(t)+\hat{\mathbf{B}}(t) \mathbf{u}(t)$

where the $m q$-dimensional $\mathbf{y}(t)$ vector is expressed as

$$
\begin{aligned}
\mathbf{y}(t) & =\left[\begin{array}{lllll}
\mathbf{x}^{\mathrm{T}}(t) & \cdots & \mathbf{x}^{\mathrm{T}}(t-\tau)
\end{array}\right]^{\mathrm{T}} \\
& =\left[\begin{array}{llllll}
\mathbf{y}_{1}^{\mathrm{T}}(t) & \mathbf{y}_{2}^{\mathrm{T}}(t) & \mathbf{y}_{3}^{\mathrm{T}}(t) & \cdots & \mathbf{y}_{m}^{\mathrm{T}}(t)
\end{array}\right]^{\mathrm{T}}
\end{aligned}
$$

where $\mathbf{y}_{\alpha}(t)=\mathbf{x}\left(t-\frac{\tau}{2}\left(1-t_{\alpha-1}\right)\right), \alpha=1, \ldots, m$. The time-varying matrices $\hat{\mathbf{A}}(t)$ (of dimension $m q \times m q$ ) and $\hat{\mathbf{B}}$ (of dimension $m q \times p$ ) in Eq. (5) are expressed as

$$
\begin{aligned}
\hat{\mathbf{A}}(t) & =\left[\begin{array}{lllll}
\mathbf{A}_{1}(t) & \mathbf{0}_{q} & \cdots & \mathbf{0}_{q} & \mathbf{A}_{2}(t) \\
& & \frac{2}{\tau}\left[\mathbb{D}_{m q \times m q}^{(q+1, m q)}\right.
\end{array}\right], \\
& \hat{\mathbf{B}}(t)=\left[\begin{array}{llll}
\mathbf{B}^{T}(t) & 0 & \cdots & 0
\end{array}\right]^{T}
\end{aligned}
$$

where $\mathbb{D}_{m q \times m q}=\mathbf{D}_{m \times m} \otimes \mathbf{I}_{q \times q}$, in which only rows of $\mathbb{D}$ between $q+1$ and $m q$ enter in the $m q \times m q$ matrix $\hat{\mathbf{A}}$ and $\otimes$ is the Kronecker product. Superscript $(q+1, m q)$ on $\mathbb{D}_{m q \times m q}$ refers to the fact that only rows of $\mathbb{D}_{m q \times m q}$ between $q+1$ and $m q$ are written into the remaining $N q \times m q$ part of matrix $\hat{\mathbf{A}}(t)$. Note that the $2 / \tau$ factor in front of the portion of $\mathbb{D}_{m q \times m q}$ above accounts for the fact of rescaling the standard collocation expansion interval $[-1,1]$ to $[0, \tau]$. The illustration for the Chebyshev collocation points is given in Fig. 1. Note that, according to [23], the spectral accuracy of the CSCTA technique is inherited from Chebyshev spectral

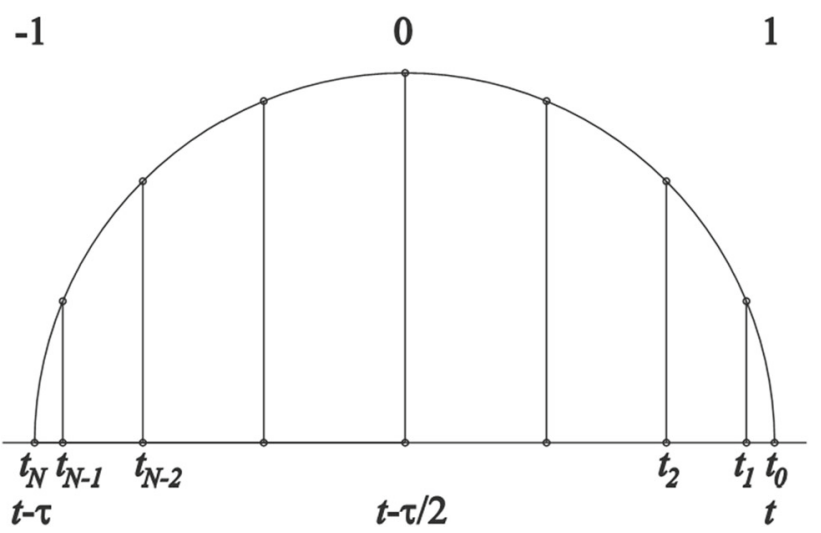

Fig. 1 Chebyshev collocation points [26] collocation. While the exponential convergence of CSCTA is numerically verified in [13], this property of Chebyshev collocation is further elaborated with details on convergence rate as $m$ increases in [24] for its use in the discretization of PDEs and in [25] for periodic DDEs specifically where it is shown that the error goes to zero as $m$ goes to infinity.

Suppose it is desired to design a linear full-state [in $\mathbf{y}(t)$ ] feedback control law with periodic control gains as

$\mathbf{u}(t)=-\mathbf{K}(t) \mathbf{y}(t)$

such that the discretized system in Eq. (5) becomes asymptotically stable where the $p \times m q$ matrix $\mathbf{K}(t)=\mathbf{K}(t+T)$ is periodic. Note this is equivalent to a delayed feedback control with distributed delay in Eq. (1) and that a large number of control gains are available, both from the discretization of the distributed delay feedback control and from the Fourier series expansion (up to any desired order) of the periodic coefficients of $\mathbf{K}(t)$, to control a large number of the closedloop poles. If only the first $q$ columns of $\mathbf{K}(t)$ are nonzero, then Eq. (7) is equivalent to a non-delayed state feedback control. Our assumption of controllability applies only to $\left[\mathbf{A}_{1}(t), \mathbf{B}(t)\right]$ in Eq. (1), because in fact the pair $[\hat{\mathbf{A}}(t), \hat{\mathbf{B}}(t)]$ in Eq. (5) may not be controllable even if the pair $\left[\mathbf{A}_{1}(t) \mathbf{B}(t)\right]$ is originally controllable as shown in [11]. Practically, this is not a problem since all we require is to control the current state $\mathbf{y}_{1}(t)=\mathbf{x}(t)$ and not $\mathbf{y}_{i}(t), i=2, \ldots, m$ as these are simply delayed states. Hence, for pole placement we typically cannot place all $m q$ poles even if the matrices $\hat{\mathbf{A}}, \hat{\mathbf{B}}$, and $\mathbf{K}$ are time-invariant. Again, this is not a concern as long as the poles being placed include the dominant ones.

We now consider three possible strategies for determining the gain matrix $\mathbf{K}(t)$ in the system. These strategies are utilized to control the response of the delayed Mathieu equation which is a prototypical representative of combined effects of time delay and parametric resonance in a dynamical system. More specifically, in all control strategies detailed below, the system under consideration is of the form

$\ddot{x}+(a+b \cos \Omega t) x(t)=c x(t-\tau)+u(t)$,

$(x, \dot{x})=(1,0), \quad-\tau \leq t \leq 0$,

where $a=0.2, b=0.1, c=0.5, \Omega=2 \pi$, and $\tau=1$. Note that the parametric period is equal to the delay. This parameter set for the uncontrolled system corresponds to the unstable point denoted by an asterisk $(*)$ in the stability diagram in Fig. 2. The matrices $\mathbf{A}_{1}, \mathbf{A}_{2}$, and $\mathbf{B}$ in Eq. (1) take the form of

$$
\begin{aligned}
\mathbf{A}_{1} & =\left[\begin{array}{cc}
0 & 1 \\
-(0.2+0.1 \cos (2 \pi t)) & 0
\end{array}\right], \quad \mathbf{A}_{2}=\left[\begin{array}{cc}
0 & 0 \\
0.5 & 0
\end{array}\right], \\
\mathbf{B} & =\left[\begin{array}{l}
0 \\
1
\end{array}\right] .
\end{aligned}
$$



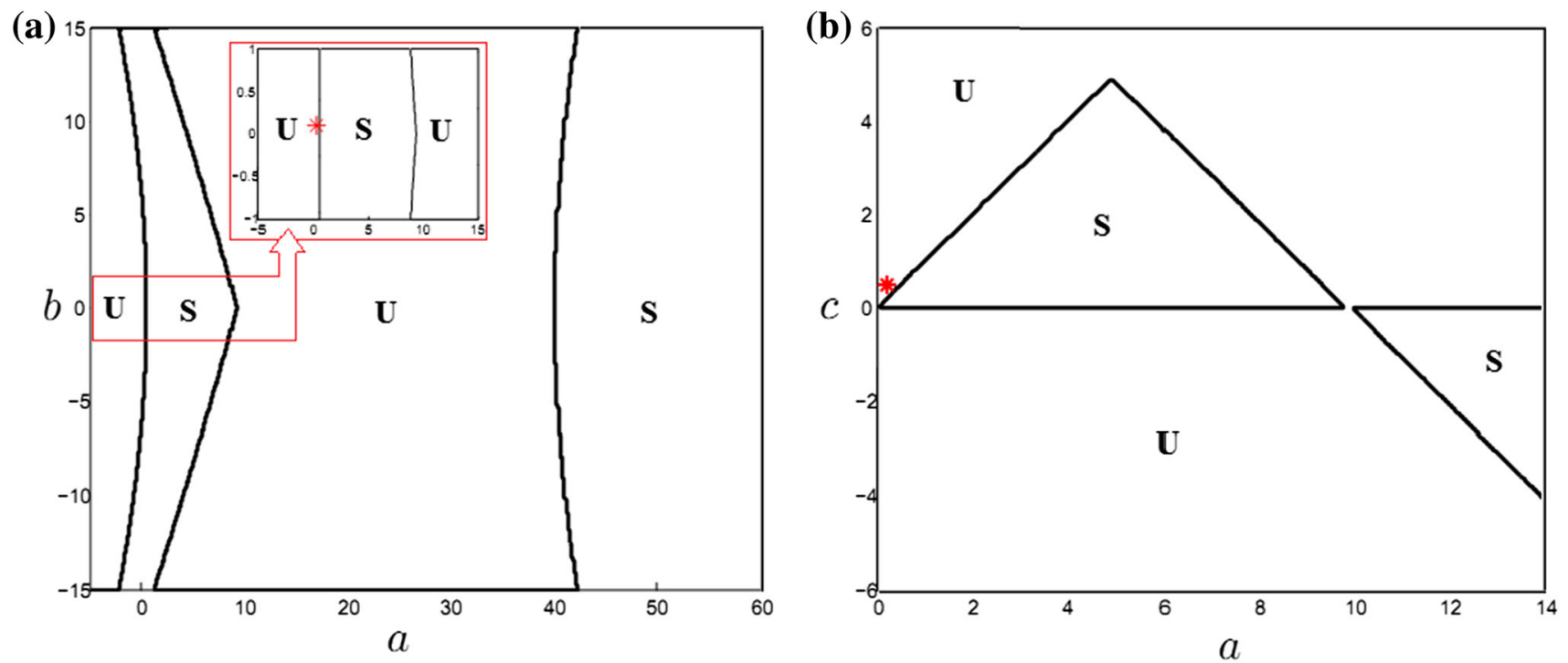

Fig. 2 The stability diagrams for the delayed Mathieu's equation given in Eq. (8) for $\mathbf{a} c=0.5$ in the $a-b$ plane and $\mathbf{b} b=0.1$ in the $a-c$ plane. The unstable point under study is indicated by asterisk

\section{Optimal control via delayed state feedback with discrete delay for minimum spectral radius}

In this strategy, which extends the delayed feedback control strategies in $[2,11,15]$ to include periodic control gains, the stability of the closed-loop response may be investigated in the parameter space of available control gains using Floquet theory, including the optimization of the control gains by minimizing the spectral radius of the monodromy operator which must be less than unity for asymptotic stability. We note that rather than obtaining the monodromy matrix associated with the closed-loop system of Eqs. (5) and (7) with system matrix $(\hat{\mathbf{A}}(t)-\hat{\mathbf{B}}(t) \mathbf{K}(t))$ by numerical integration over one period, other numerical techniques such as Chebyshev collocation [14] and semidiscretization [2] may be directly applied to the closed-loop DDE to more efficiently generate an approximation to the infinite-dimensional monodromy operator. This was employed in [15] using semidiscretization as well as in [3] via Chebyshev polynomial expansion.

An infinite dimensional dynamic map can be defined for the closed-loop system as [14]

$\mathbf{m}_{\mathbf{x}}(i)=\mathcal{U} \mathbf{m}_{\mathbf{x}}(i-1)$

that maps the state $\mathbf{x}$ in the time interval $[-\tau, 0]$ to that in $[0, \tau]$, and subsequently to the other periods after that. In Eq. (10), $\mathbf{m}_{\mathbf{x}}$ is an expansion of the state $\mathbf{x}$ in some basis which satisfies the initial condition of $\mathbf{m}_{\mathbf{x}}(0)=\mathbf{m}_{\phi}$, where $\mathbf{m}_{\phi}$ is the expansion of $\phi(\theta)$, and $\mathcal{U}$ is the infinite dimensional monodromy operator. All eigenvalues of $\mathcal{U}$ must lie inside the unit circle in the complex plane for the system to be stable. To approximate $\mathcal{U}$ by a matrix of finite dimension, Chebyshev collocation method [14] is used and hence the $\mathbf{m}_{\mathbf{x}}(i)$ and $\mathbf{m}_{\mathbf{x}}(i-1)$ are $m q$-dimensional. Using the Chebyshev collocation expansion, Eq. (1) with the delayed feedback control law $\mathbf{u}(t)=-\mathbf{K}(t) \mathbf{x}(t-\tau)$ can be expressed algebraically as

$\hat{\mathbf{D}} \mathbf{m}_{\mathbf{x}}(i)=\hat{\mathbf{M}}_{\mathbf{A}_{1}} \mathbf{m}_{\mathbf{x}}(i)+\hat{\mathbf{M}}_{\mathbf{A}_{2}-\mathbf{B K}} \mathbf{m}_{\mathbf{x}}(i-1)$

where

$$
\begin{aligned}
\mathbf{m}_{\mathbf{x}}(i) & =\left[\mathbf{x}^{T}\left(t_{0}\right), \mathbf{x}^{T}\left(t_{1}\right), \cdots, \mathbf{x}^{T}\left(t_{N}\right)\right]^{T}, \\
\mathbf{m}_{\mathbf{x}}(i-1) & =\left[\phi^{T}\left(t_{0}\right), \phi^{T}\left(t_{1}\right), \cdots, \phi^{T}\left(t_{N}\right)\right]^{T}
\end{aligned}
$$

and the matrix

$\hat{\mathbf{D}}=\left[\begin{array}{cccc} & & \frac{2}{\tau}[\mathbb{D}]^{(m-1) q, m q} & \\ \mathbf{0}_{q} & \mathbf{0}_{q} & \cdots & \mathbf{I}_{q}\end{array}\right]$

accounts for the derivation of the state. The monodromy operator $\mathcal{U}$ is therefore approximated by

$\mathbf{U}=\left[\hat{\mathbf{D}}-\hat{\mathbf{M}}_{\mathbf{A}_{1}}\right]^{-1} \hat{\mathbf{M}}_{\mathbf{A}_{2}-\mathbf{B K}}$

where

$$
\hat{\mathbf{M}}_{\mathbf{A}_{1}}=\left[\begin{array}{ccccc}
\mathbf{A}_{1}\left(t_{0}\right) & & & & \\
& \mathbf{A}_{1}\left(t_{1}\right) & & & \\
& & \ddots & & \\
& & & \mathbf{A}_{1}\left(t_{N-1}\right) & \\
\mathbf{0}_{q} & \mathbf{0}_{q} & \mathbf{0}_{q} & \cdots & \mathbf{0}_{q}
\end{array}\right] \text {, }
$$

$\hat{\mathbf{M}}_{\mathbf{A}_{2}-\mathbf{B K}}$

$$
=\left[\begin{array}{ccccc}
\left(\mathbf{A}_{2}-\mathbf{B K}\right)\left(t_{0}\right) & & & & \\
& \left(\mathbf{A}_{2}-\mathbf{B K}\right)\left(t_{1}\right) & & & \\
& & \ddots & & \\
& & & \left(\mathbf{A}_{2}-\mathbf{B K}\right)\left(t_{N-1}\right) & \\
\mathbf{I}_{q} & \mathbf{0}_{q} & \mathbf{0}_{q} & \ldots & \mathbf{0}_{q}
\end{array}\right] .
$$


The approximated monodromy operator $\mathbf{U}$ of the uncontrolled system is found to have a spectral radius larger than 1 and hence the uncontrolled system is unstable. In order to obtain the asymptotic stability of the controlled system, we design a delayed state feedback controller with discrete delay and a periodic gain matrix in which each gain component is expressed in a 3-term Fourier series with unknown coefficients as

$$
\begin{aligned}
u(t)= & -\left(k_{11}+k_{12} \cos 2 \pi t+k_{13} \sin 2 \pi t\right) x(t-1) \\
& -\left(k_{21}+k_{22} \sin 2 \pi t+k_{23} \cos 2 \pi t\right) \dot{x}(t-1)
\end{aligned}
$$

Note that the parametric period and delay $(T=\tau=1)$ in Eq. (16) are the same as in Eq. (8). The difficulty of obtaining the measurements of derivatives in a noisy environment is addressed in $[27,28]$, but is not considered here.

First, we set $k_{12}=k_{13}=k_{22}=k_{23}=0$ and find the stable region in the $k_{11}-k_{21}$ parameter space for constant gain delayed feedback. For this purpose, the monodromy operator $\mathbf{U}$ that governs the period-to-period mapping of the initial function $\phi(t)$ is approximated using the Chebyshev collocation method. The set of the points in the $k_{11}-k_{21}$ parameter space for which the spectral radius of $\mathbf{U}$ lies on the circumference of the unit circle forms the stability boundary. Starting from a unit spectral radius and plotting contours for gradually decreasing spectral radii, the $\left(k_{11}, k_{21}\right)$ location corresponding to the minimum spectral radius can be obtained. The optimum set of the first pair of the control gains is $\left(k_{11}, k_{21}\right)=(0.40,0.35)$ which corresponds to the spectral radius $\rho=0.54577$. Next, the optimal values obtained for $k_{11}$ and $k_{21}$ are held constant with $k_{13}=k_{23}=0$ while the stable region in the $k_{12}-k_{22}$ parameter space is found as well as the values of $k_{12}$ and $k_{22}$ that correspond to the minimum spectral radius for the new controlled system. The optimum set of the second pair of the control gains, i.e. $\left(k_{12}, k_{22}\right)=(-0.33,-0.06)$, corresponds to the spectral radius $\rho=0.52154$. Finally, the optimum values obtained for $k_{11}, k_{21}, k_{12}$, and $k_{22}$ are held constant while the stability region in the $k_{13}-k_{23}$ parameter space is found as well as their optimum values. The minimum spectral radius is $\rho_{\text {opt }}=0.51977$ which corresponds to $\left(k_{13}, k_{23}\right)=(0.03,-0.06)$. Altogether, the opti$\mathrm{mal}$ set of the control gains is $\left(k_{11}, k_{21}, k_{12}, k_{22}, k_{13}, k_{23}\right)=$ $(0.40,0.35,-0.33,-0.06,0.03,-0.06)$. These results are obtained using 150 Chebyshev collocation points and a $500 \times 500$ meshgrid for each parameter space. Upon iterative optimization of the control gains, the results obtained are found to be converged up to the second decimal place. Therefore, one can say that the aforementioned optimal set of the control gains is locally optimal, while further examination of the entire control gain space reveals this is also the global optimum for the control law of Eq. (16). The optimum control gains for minimum spectral radius are denoted with a square (filled square) in the stability diagrams shown in Fig. 3. Figure 4 shows the uncontrolled response of the system in Eq. (8), the controlled response using the optimal constant control law, and that using the optimal full 6-term periodic control law, along with the corresponding control efforts. The total control efforts for these control laws are $\int_{0}^{t_{f}}|u(\eta)| d \eta=$ 1.7708 and 1.7792 , respectively. The simulated results indicate that when the optimal values of the control gains are used, the closed-loop response has less settling time due to a greater effective damping, and that $k_{11}$ and $k_{21}$ have a more significant role in the convergence speed. Note that the use of additional Fourier terms in the control law could further reduce the spectral radius of the closed-loop response.

Note that although semidiscretization [2] can also be utilized for the optimum control design, because of the spectral convergence in the Chebyshev collocation method, we have only shown the results obtained by the latter method, although identical results could be obtained using a sufficiently large meshgrid in the semidiscretization technique.

\section{Optimal control via delayed state feedback with distributed delay using CSCTA and time-varying LQR}

The second control strategy we consider utilizes CSCTA but not RLFT. First, Eq. (8) which is in the form of Eq. (1) is discretized using CSCTA and is written in the form of Eq. (5). Then, optimal (time-varying LQR) control is used to minimize the cost function of the resulting discretized equation, i.e.

$$
\begin{aligned}
J= & \frac{1}{2} \mathbf{y}^{T}\left(t_{f}\right) \mathbf{S y}\left(t_{f}\right) \\
& +\frac{1}{2} \int_{0}^{t_{f}}\left(\mathbf{y}^{T}(t) \mathbf{Q}(t) \mathbf{y}(t)+\mathbf{u}^{T}(t) \mathbf{R}(t) \mathbf{u}(t)\right) d t
\end{aligned}
$$

where $\mathbf{Q}(t) \geq \mathbf{0}$ and $\mathbf{R}(t)>\mathbf{0}$ are $T$-periodic weight matrices and $\mathbf{S} \geq \mathbf{0}$ is the matrix of the terminal cost. The control law is given by Eq. (7) where the gain matrix is found from standard LQR theory by solving the PRE [29]

$$
\begin{aligned}
\dot{\mathbf{P}}(t)= & -\left(\hat{\mathbf{A}}^{T}(t) \mathbf{P}(t)+\mathbf{P}(t) \hat{\mathbf{A}}(t)+\mathbf{Q}(t)\right) \\
& +\mathbf{P}(t) \hat{\mathbf{B}}(t) \mathbf{R}^{-1}(t) \hat{\mathbf{B}}^{T}(t) \mathbf{P}(t)
\end{aligned}
$$

for $\mathbf{P}(t)=\mathbf{P}(t+T)$ backwards in time from the final value $\mathbf{P}\left(t_{f}\right)=\mathbf{S}$ or by solving the periodic ARE [30]

$$
\begin{gathered}
-\left(\hat{\mathbf{A}}^{T}(t) \mathbf{P}(t)+\mathbf{P}(t) \hat{\mathbf{A}}(t)+\mathbf{Q}(t)\right) \\
+\mathbf{P}(t) \hat{\mathbf{B}}(t) \mathbf{R}^{-1}(t) \hat{\mathbf{B}}^{T}(t) \mathbf{P}(t)=\mathbf{0} .
\end{gathered}
$$

Note that Eq. (19) can be efficiently solved for a uniform grid of times $t_{i} \in[0, T]$. Alternatively, the elements of $\mathbf{P}(t)$ can 

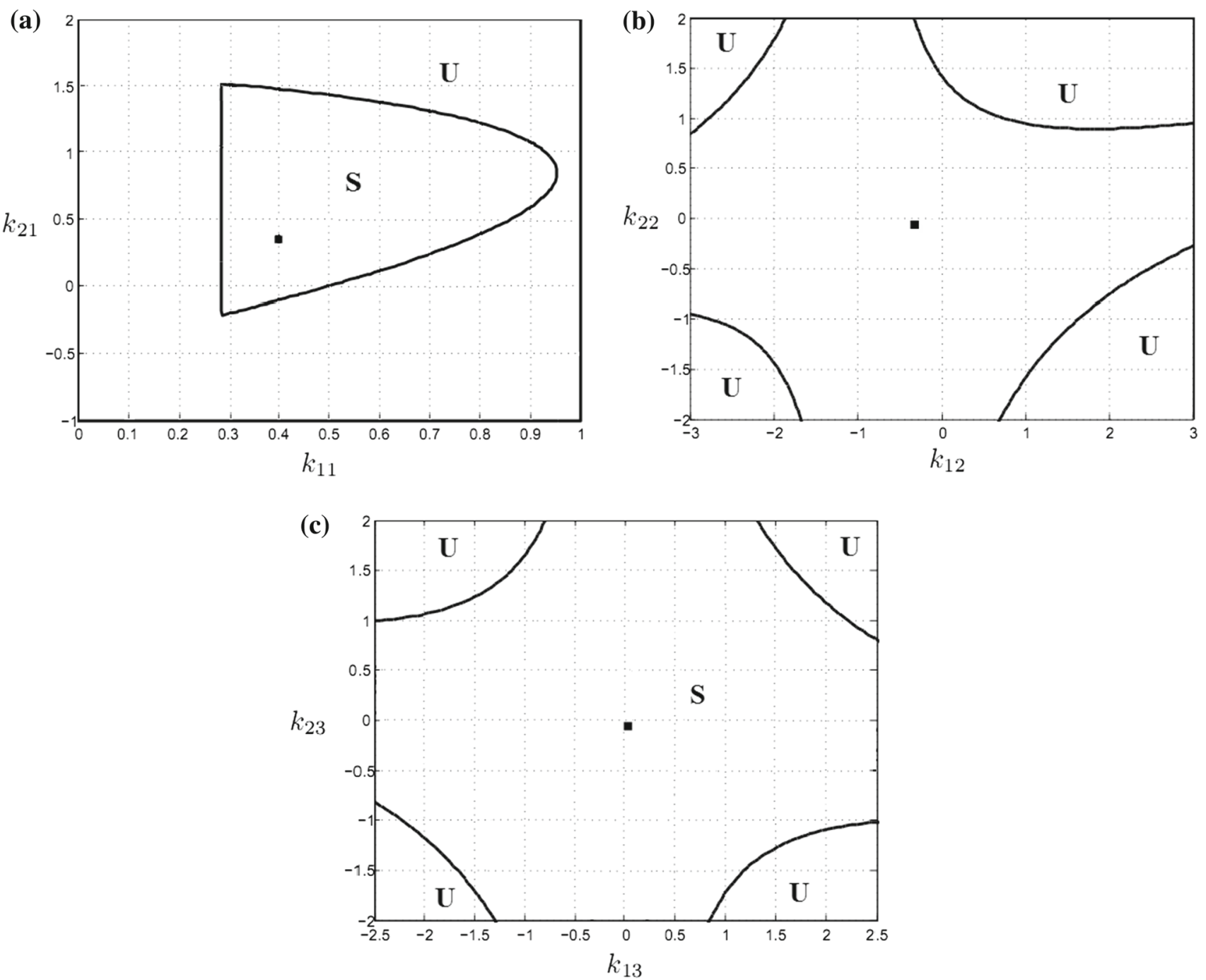

Fig. 3 The stability of the originally unstable test point asterisk shown in Fig. 2 for different combinations of the control gains. a $k_{12}=k_{22}=$ $k_{13}=k_{23}=0, \mathbf{b}\left(k_{11}, k_{21}\right)=(0.4,0.35)$ and $k_{13}=k_{23}=0$, and c $\left(k_{11}, k_{21}\right)=(0.4,0.35)$ and $\left(k_{12}, k_{22}\right)=(-0.33,-0.06)$. The sta-

be expanded in a Fourier series with an arbitrary number of terms and the Fourier coefficients obtained by solving systems of nonlinear algebraic equations resulting from either Eq. (18) or (19), where additional Fourier terms results in further optimization of Eq. (17). Note, however, that solving for Fourier coefficients is computationally intensive and may not be practical in applications. The periodic control gain is then obtained through the well-known equation [29]

$\mathbf{K}(t)=\mathbf{R}^{-1}(t) \hat{\mathbf{B}}^{T}(t) \mathbf{P}(t)$,

where $\mathbf{P}(t)$ is either the solution of the PRE (18) or ARE (19).

In this example, the final condition is assumed to be $\mathbf{P}\left(t_{f}\right)=\mathbf{S}=\mathbf{0}$ and $m=11$ Chebyshev collocation points are used. The weighting matrices are selected such that $\mathbf{Q}=$ $\alpha \mathbf{I}_{m q \times m q}$ (first case) and $\mathbf{Q}=\operatorname{diag}\left[\alpha \mathbf{I}_{q \times q}, \mathbf{0}_{(m q-q) \times(m q-q)}\right]$ ble $(\mathrm{S})$ and unstable $(\mathrm{U})$ regions are indicated in different parameter spaces, and the gain pair for minimum spectral radius of the closedloop response is shown with filled square in each diagram

(second case) in the simulations, where different values $(\in[0,16])$ are assigned for $\alpha$, and $\mathbf{R}=1$ is scalar. The entries $P_{11}, P_{12}$, and $P_{22}$ of the solution matrix $\mathbf{P}(t)$ and all entries of the control gain matrix $\mathbf{K}(t)$, the controlled response, and control input $u(t)$ of the system given in Eq. (8) are plotted in Figs. 5 and 6 for $\alpha=6$ and $\mathbf{R}=1$. According to the figure, the response for the case that only the top left $2 \times 2$ diagonal of the $\mathbf{Q}$ matrix contains nonzero values approaches the steady state solution (zero) slightly slower than the case that $\mathbf{Q}$ is the identity matrix, and they have almost the same total control effort. However, as expected, using the normalized cost function $J /\|\mathbf{Q}\|$, where $\|\mathbf{Q}\|$ denotes the trace norm of $\mathbf{Q}$, it is found that the cost for the second case (2.077) is less than that for the first case (2.427). The total control efforts $\int_{0}^{t_{f}}|u(\eta)| d \eta$ in Fig. 6 are 0.986 and 0.810 for the first and the 

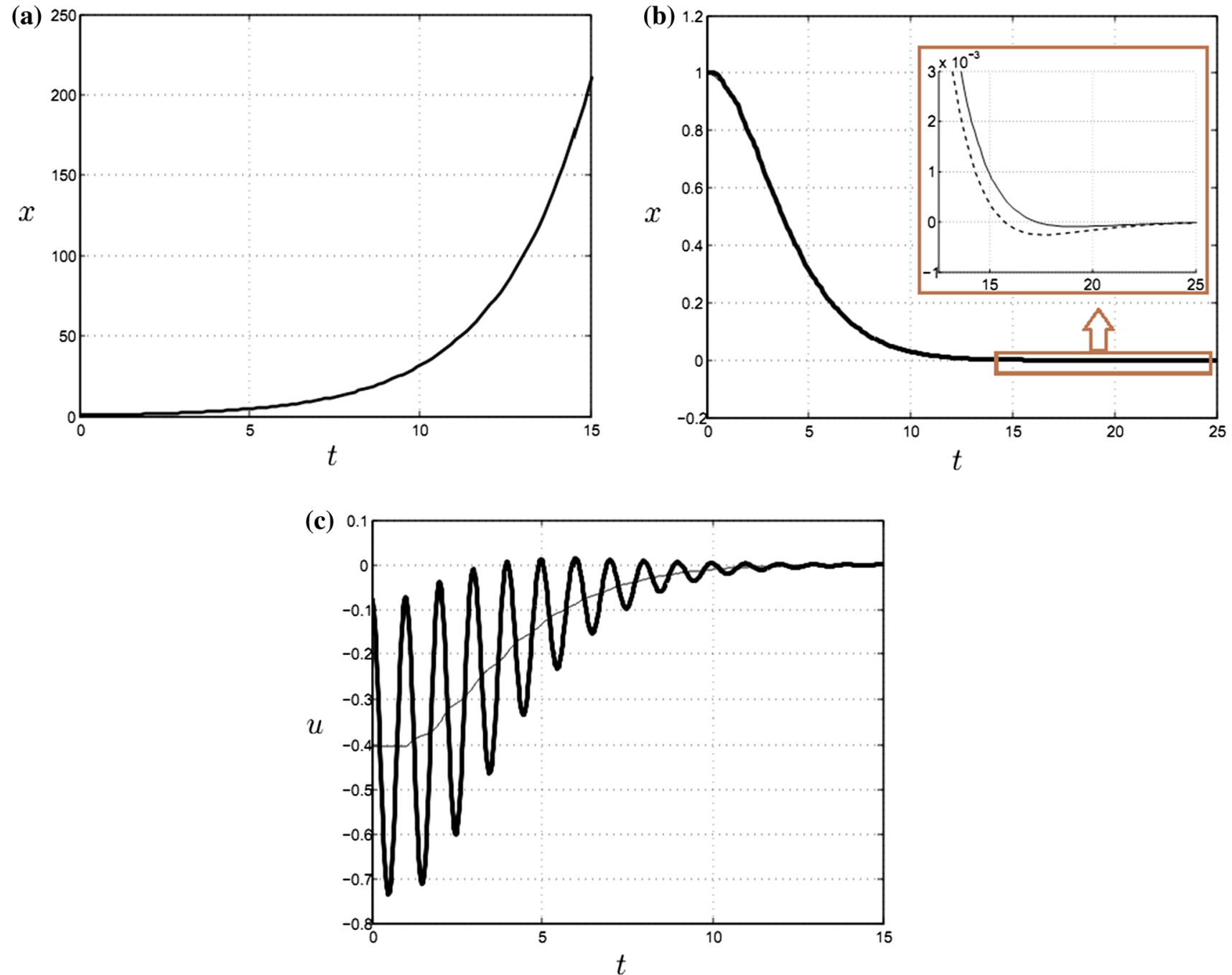

Fig. 4 a the uncontrolled response for Eq. (8), b the controlled response for Eq. (8) with the constant gain control law (dash) compared to that with the full 6-term periodic control law (solid), and $\mathbf{c}$ the control effort corresponding to the constant gain control law (thin line) compared to that corresponding to the full 6-term periodic control law (thick line) second cases, respectively. Recall that the time-varying LQR method is applied to the system after discretization using CSCTA.

To solve the periodic ARE in this study, the time was discretized into 100 intervals and the ARE was solved for each time interval. The entries $P_{11}, P_{12}$, and $P_{22}$ of the solution matrix $\mathbf{P}(t)$, all entries of the control gain matrix $\mathbf{K}(t)$, the controlled response, and control input $\mathbf{u}(t)$ of the system given in Eq. (8) are plotted in Figs. 7 and 8 for the same values of $\alpha$ and $\mathbf{R}$ as those in Figs. 5 and 6. According to Fig. 7, as with the results obtained by integrating the PRE, the response for the second case approaches the steady state solution (zero) slower than the first case and they have almost the same total control effort. However, the cost function for the second case is less than that for the first case. The normalized cost functions are 4.4479 and 4.1776 for the first and the second cases, respectively. The total control efforts are 0.9697 and 0.891 for the first and the second cases, respectively. As can be seen from Figs. 5 and 7, the steady state responses for the elements of the solution matrix $\mathbf{P}(t)$ and the control gain $\mathbf{K}(t)$ are almost the same regardless of whether the PRE given in Eq. (18) is integrated or its analogous ARE is solved. As can be seen in Figs. 6 and 8, the responses obtained via the PRE and ARE are almost identical since the controlled response is practically zero at the time when the control gains in Fig. 5 start to collapse in order to satisfy the terminal constraint. It should be mentioned that if the ARE is solved, the controller obtained is only optimal in the steady state regime. The results for $\alpha=6$ and $\mathbf{R}=1$ are compared in Fig. 9 for ARE and PRE. According to the results obtained by integrating the PRE given in Eq. (18) and solving the analogous ARE for a range of values of $\alpha \in[0,16]$ (see Fig. 10), 

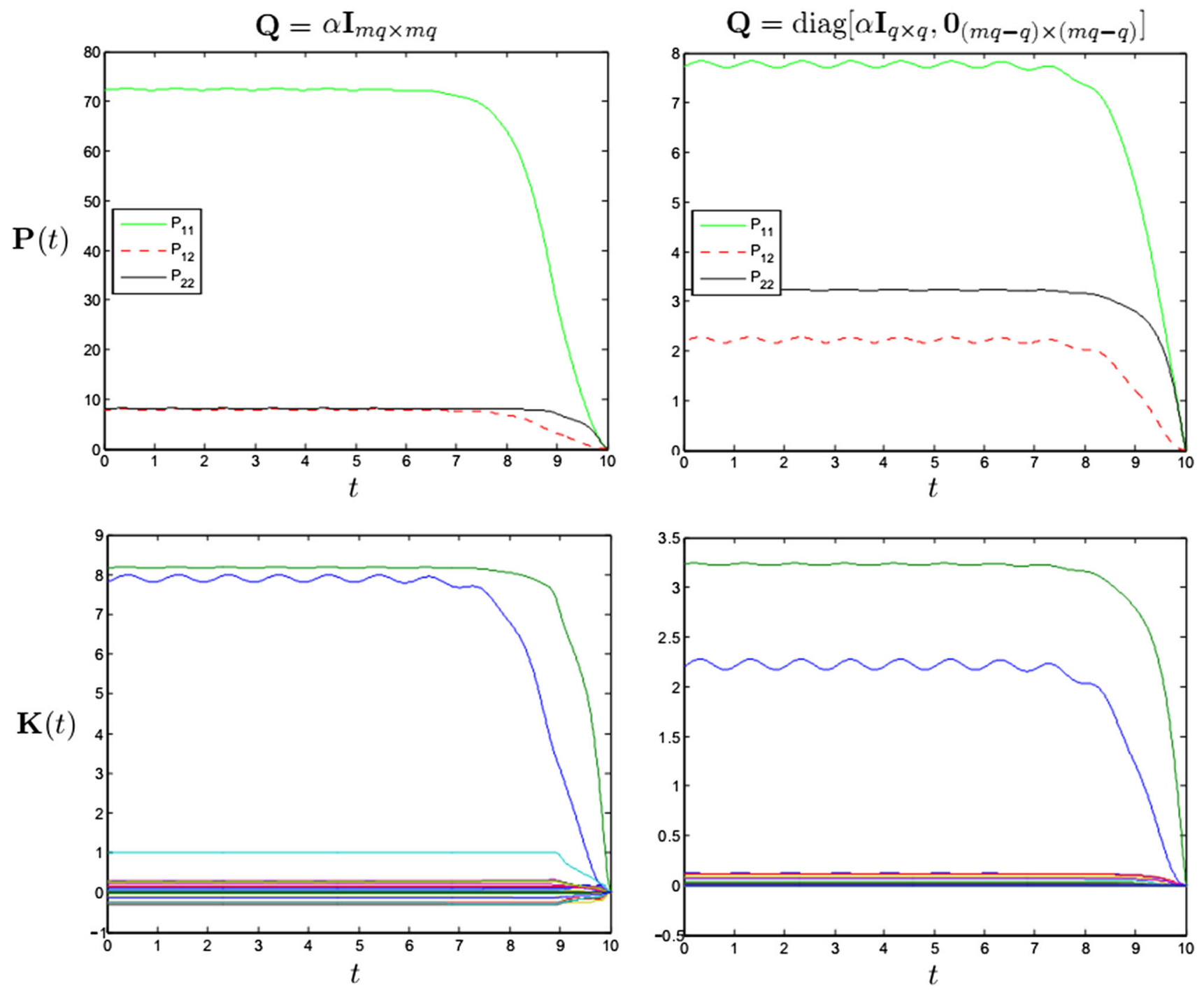

Fig. 5 The entries $P_{11}, P_{12}, P_{22}$ of the solution matrix $\mathbf{P}(t)$ (first row) and all $m q=22$ entries of the control gain matrix $\mathbf{K}(t)$ (second row) of the system given in Eq. (8) obtained by solving the PRE given in

one can conclude that integrating the PRE given in Eq. (18) for the second case with $\alpha \approx 6$ yields the minimum total control effort of $\int_{0}^{t_{f}}|u(\eta)| d \eta=0.810$.

Note that this control strategy is equivalent to delayed feedback control with distributed delay in Eq. (1), and hence the control law in Eq. (7) can be rewritten as

$\mathbf{u}(t)=-\int_{-\tau}^{0} \kappa(\theta, t) \mathbf{x}(t+\theta) d \theta$,

where $\boldsymbol{\kappa}(\theta, t)=\left[\kappa_{1}(\theta, t), \kappa_{2}(\theta, t)\right]$ is the time-periodic kernel of the distributed delay and $\mathbf{x}(t)=[x(t), \dot{x}(t)]^{T}$, in which case Eq. (1) becomes a set of DDEs with time-periodic coefficients and discrete and distributed delays, i.e.
Eq. (18) backward in time using step by step integration for the first case (left) and the second case (right). In the simulations, $\alpha=6$ and $\mathbf{R}=1$

$$
\begin{aligned}
& \dot{\mathbf{x}}(t)= \mathbf{A}_{1}(t) \mathbf{x}(t)+\mathbf{A}_{2}(t) \mathbf{x}(t-\tau) \\
&-\mathbf{B}(t) \int_{-\tau}^{0} \kappa(\theta, t) \mathbf{x}(t+\theta) d \theta, \\
& \mathbf{x}(t)=\phi(t), \quad-\tau \leq t \leq 0 .
\end{aligned}
$$

The relation between control gain matrices $\mathbf{K}(t)$ and $\boldsymbol{\kappa}(\theta, t)$ is illustrated in Fig. 11 for the delayed Mathieu equation given in Eq. (8) with $\mathbf{R}=1, \mathbf{Q}=\operatorname{diag}\left[\alpha \mathbf{I}_{q \times q}, \mathbf{0}_{(m q-q) \times(m q-q)}\right]$, $\alpha=6$, and $m=11$ Chebyshev collocation points, where $\kappa_{1}()$ and $\kappa_{2}()$ correspond to the position $x$ and velocity $\dot{x}$ of the state vector $\mathbf{x}$. According to the figure, the controller contains almost no feedback of the delayed velocity but only the current velocity, and while the current position is weighted 

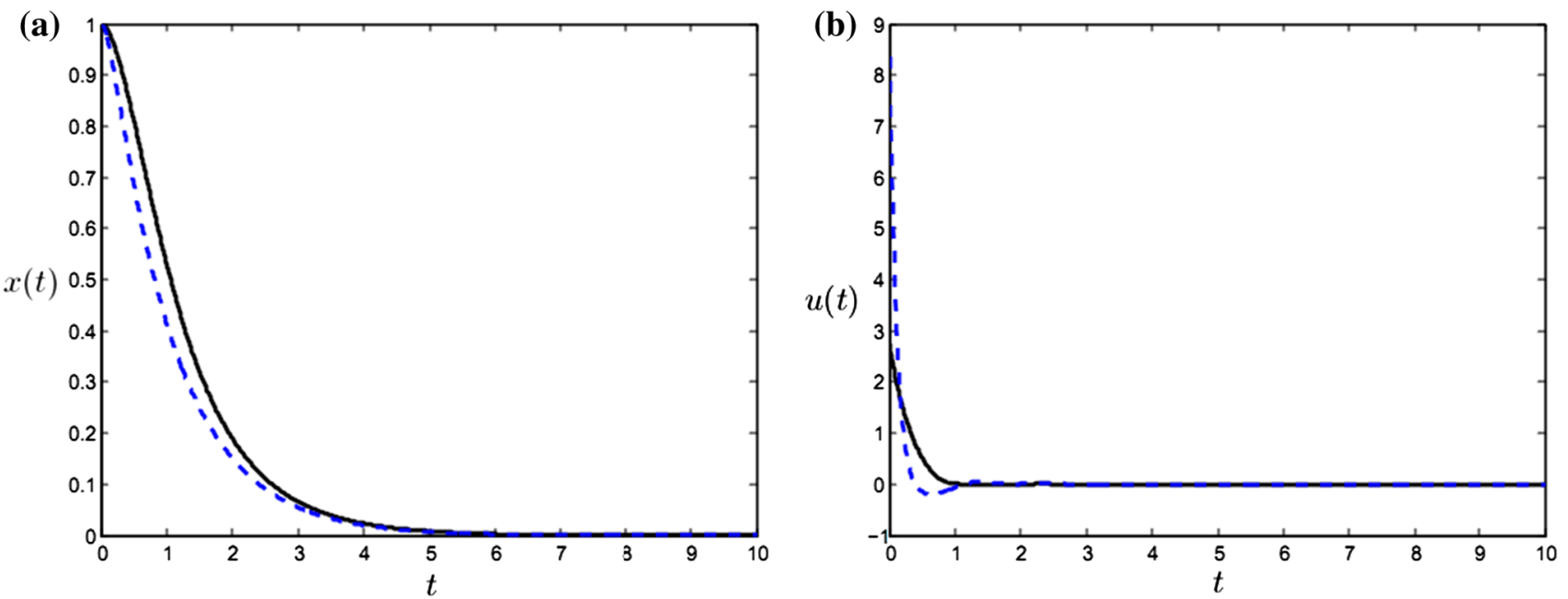

Fig. 6 The controlled response $x(t)$ (left) and control effort $u(t)$ (right) of the system given in Eq. (8) obtained by solving the PRE in Eq. (18) backward in time for the first case (dash) and the second case (solid). In the simulations, $\alpha=6$ and $\mathbf{R}=1$
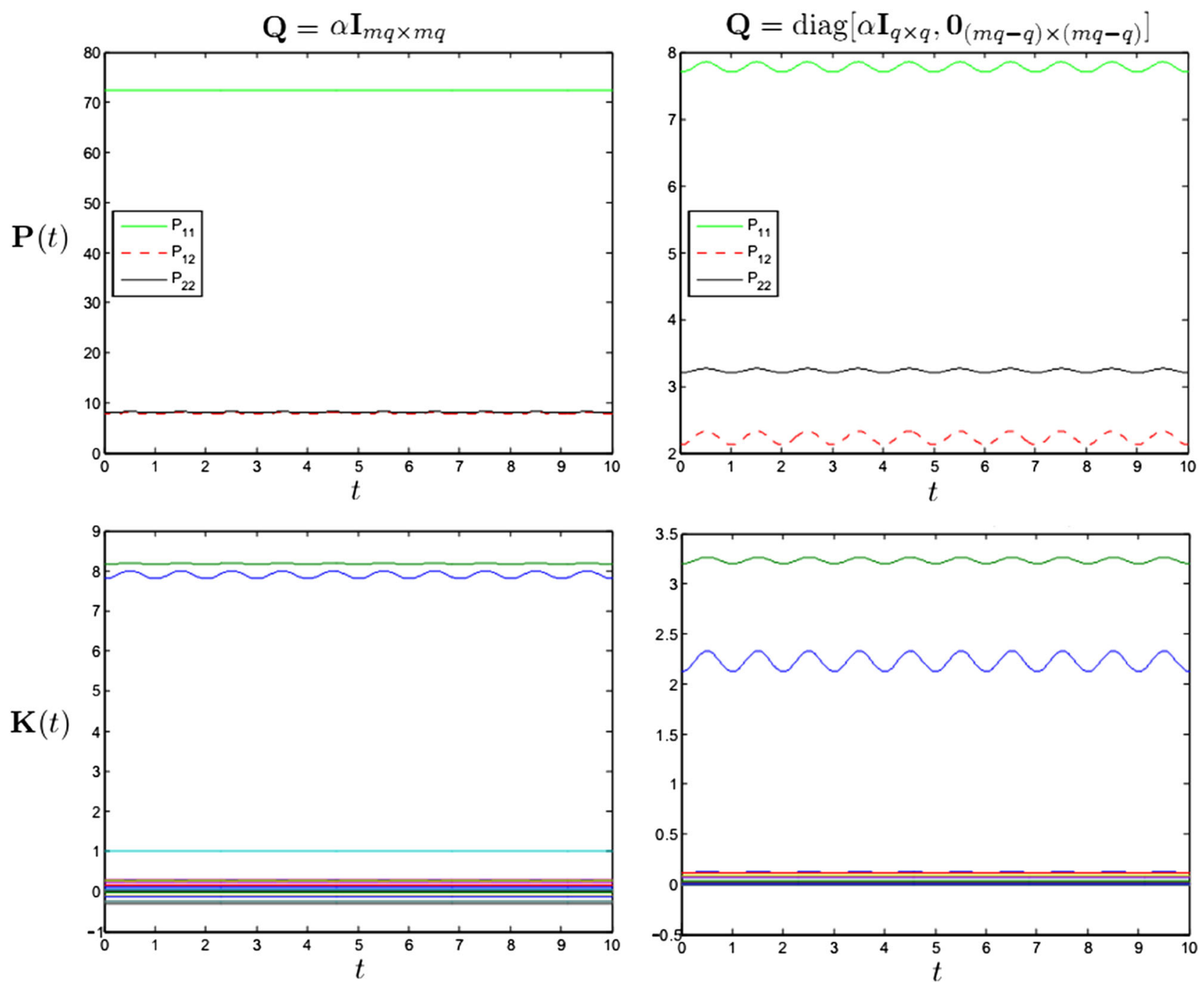

Fig. 7 The entries $P_{11}, P_{12}, P_{22}$ of the solution matrix $\mathbf{P}(t)$ (first row) and all $m q=22$ entries of the control gain matrix $\mathbf{K}(t)$ (second row) of the system given in Eq. (8) obtained by solving the ARE in Eq. (19) for the first case (left) and the second case (right). In both simulations, $\alpha=6$ and $\mathbf{R}=1$ 

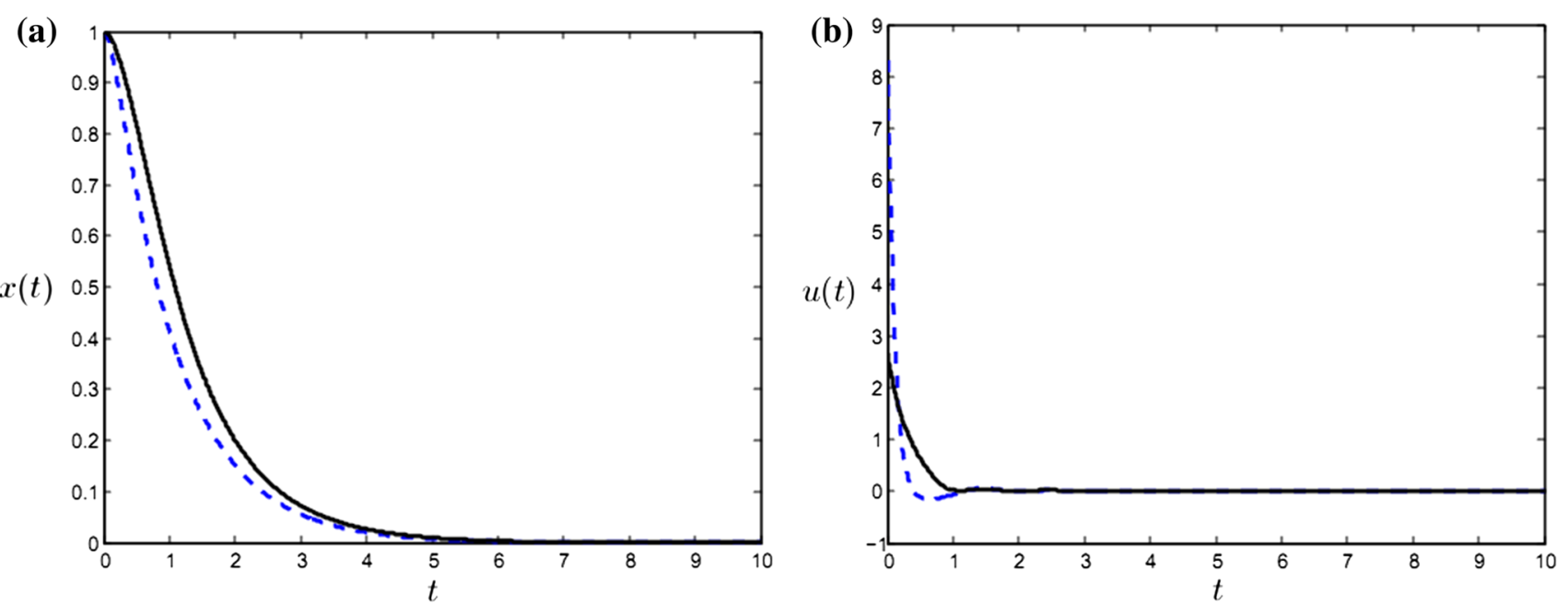

Fig. 8 The controlled response $x(t)$ (left) and control effort $u(t)$ of the system given in Eq. (8) obtained by solving the ARE in Eq. (19) for the first case (dash) and the second case (solid). In both simulations, $\alpha=6$ and $\mathbf{R}=1$
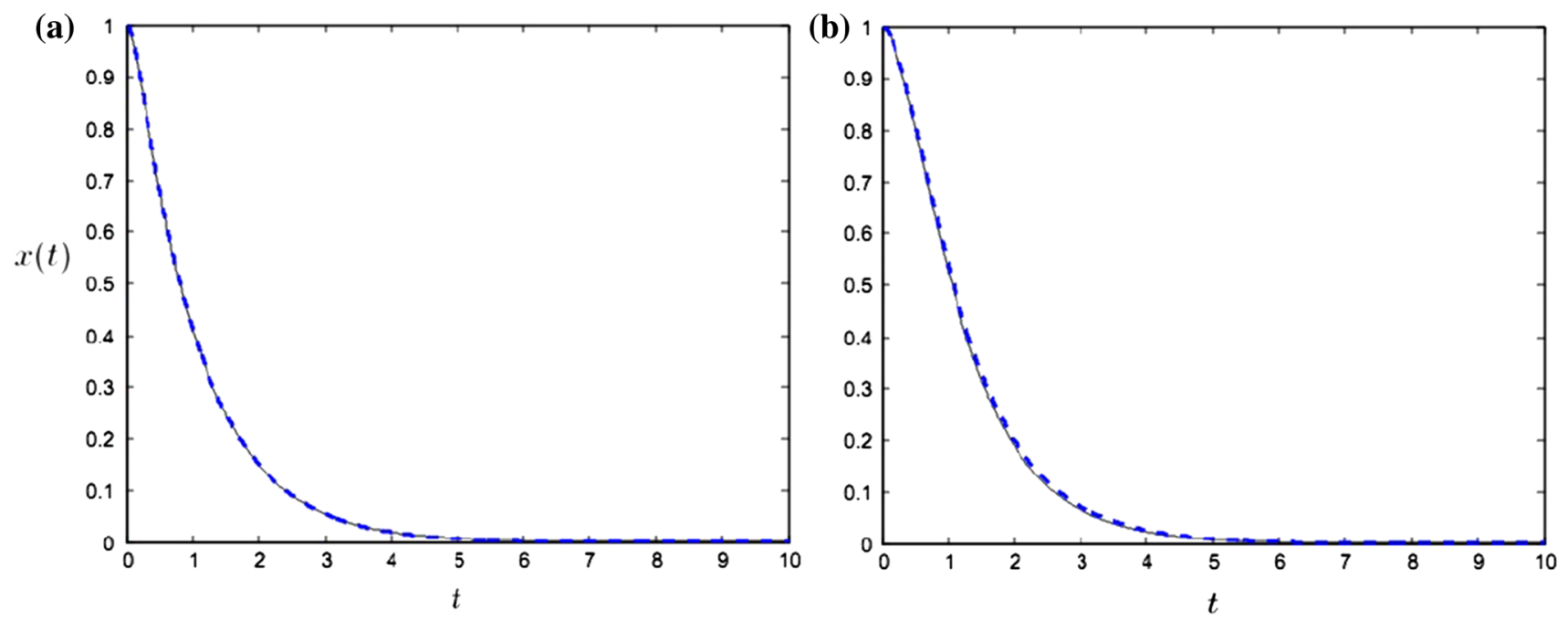

Fig. 9 The controlled response of the system given in Eq. (8) obtained by integrating the PRE in Eq. (18) backward in time (solid) compared to that obtained by solving the ARE in Eq. (19) (dash) for $\mathbf{a}$ the first case and $\mathbf{b}$ the second case. In the simulations, $\alpha=6$ and $\mathbf{R}=1$

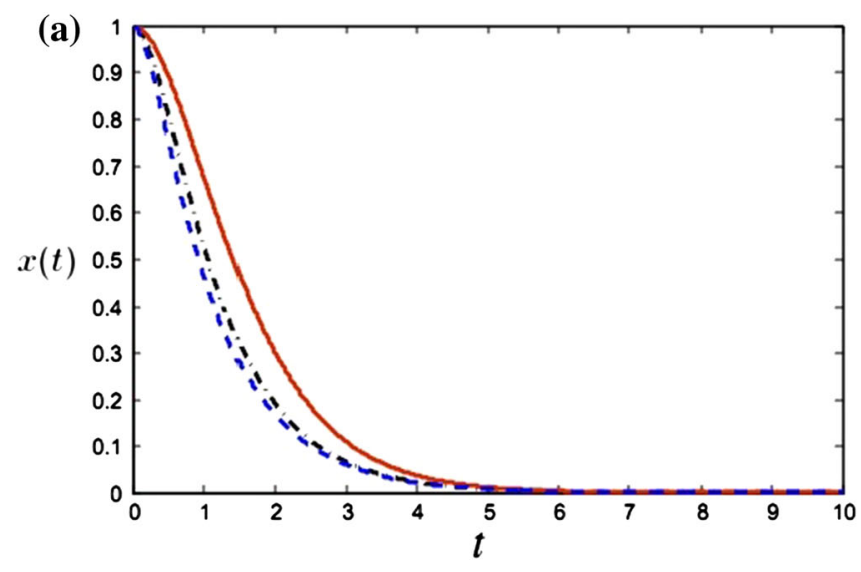

Fig. 10 a The controlled response of the system given in Eq. (8) obtained by integrating the PRE in Eq. (18) backward in time when $\mathbf{Q}=\operatorname{diag}\left[\alpha \mathbf{I}_{q \times q}, \mathbf{0}_{(m q-q) \times(m q-q)}\right] . \alpha=1$ (solid), $\alpha=6$ (dashdot), and $\alpha=16$ (dash); b The total control effort versus $\alpha$ when

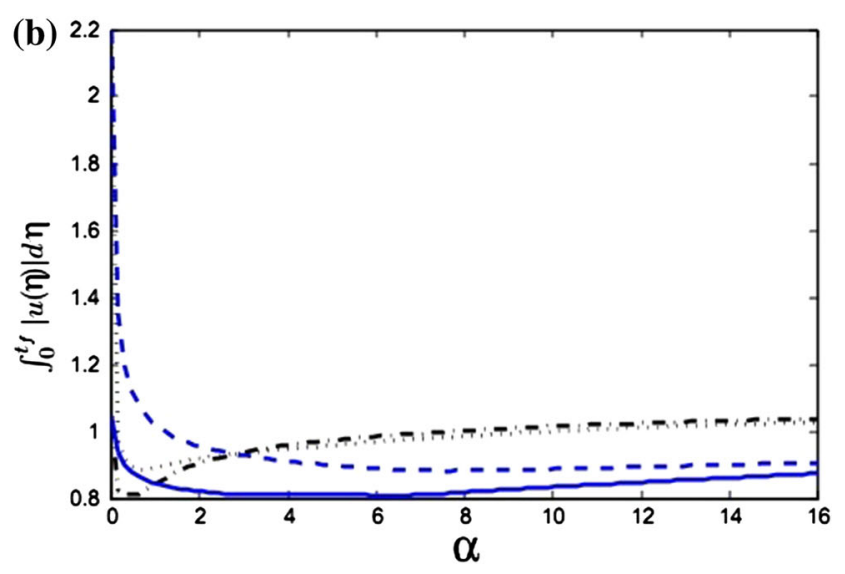

$\mathbf{Q}=\alpha \mathbf{I}_{m q \times m q}$ obtained by solving PRE (dash-dot) and ARE (dot), and when $\mathbf{Q}=\operatorname{diag}\left[\alpha \mathbf{I}_{q \times q}, \mathbf{0}_{(m q-q) \times(m q-q)}\right]$ obtained by solving PRE (solid) and ARE (dash). In the simulations, $\mathbf{R}=1$ 

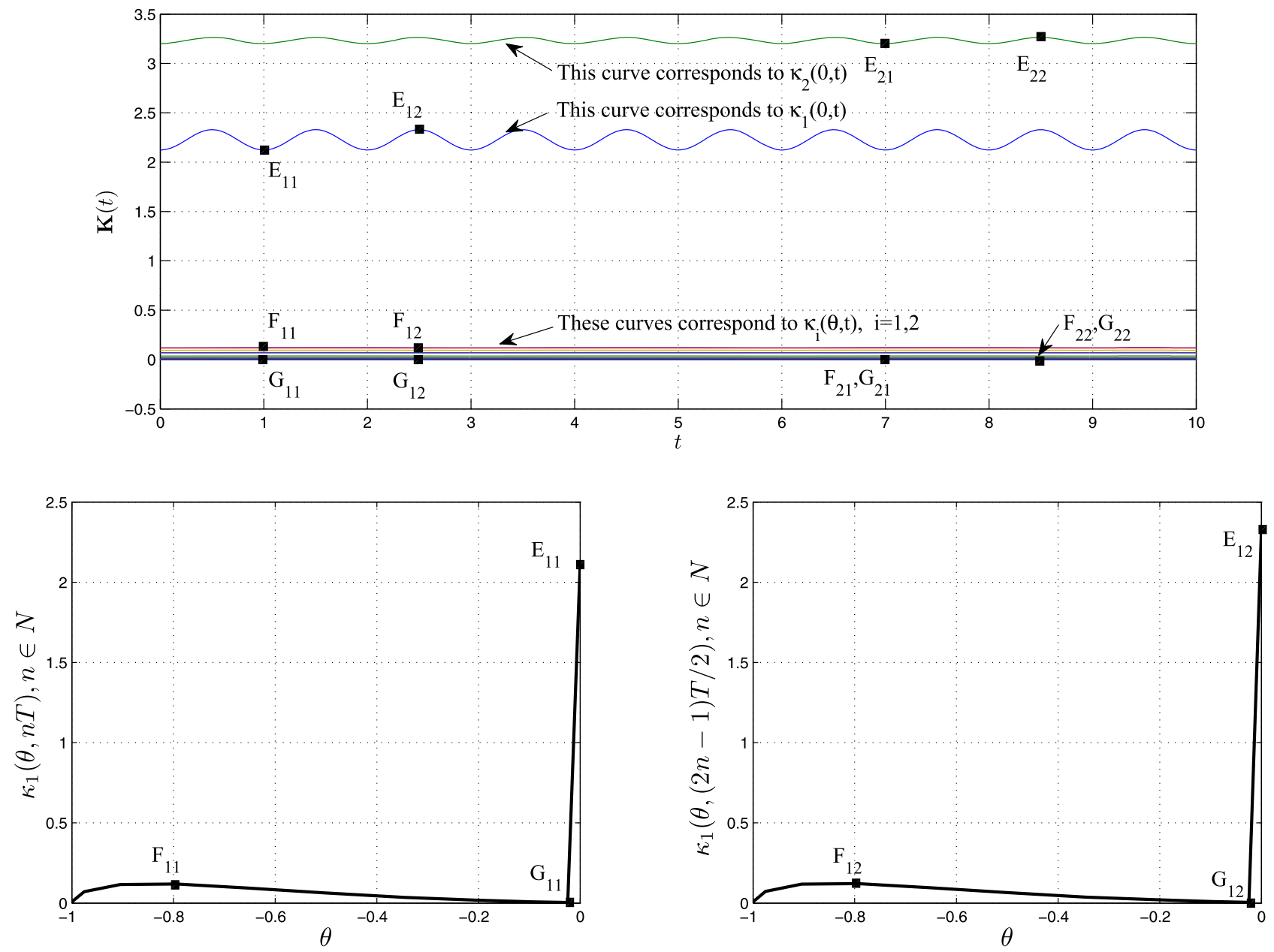

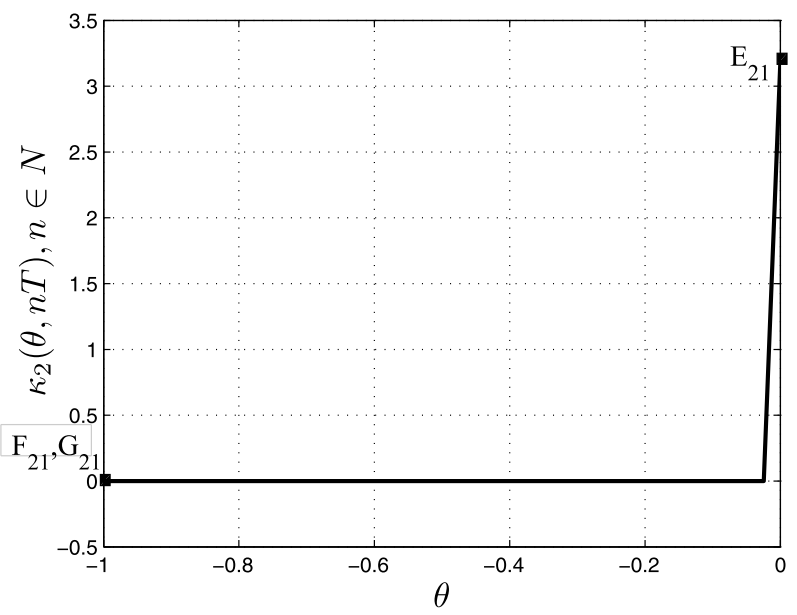

Fig. 11 Control gain matrix $\mathbf{K}(t)$ obtained from solving the periodic ARE with $\mathbf{R}=1, \mathbf{Q}=\operatorname{diag}\left[\alpha \mathbf{I}_{q \times q}, \mathbf{0}_{(m q-q) \times(m q-q)}\right], \alpha=6$, and $m=11$ Chebyshev collocation points (top) versus the equivalent control gain kernel $\kappa(\theta, t)=\left[\kappa_{1}(\theta, t), \kappa_{2}(\theta, t)\right]$ of the distributed delayed

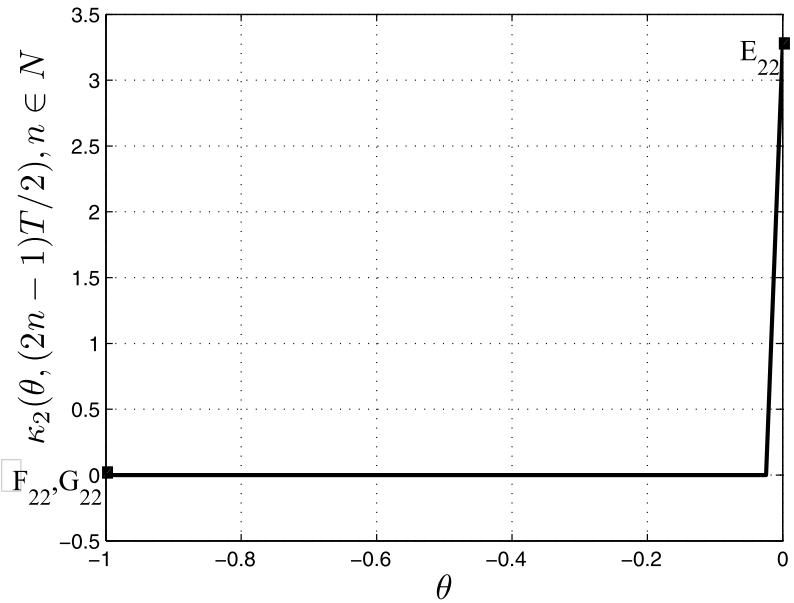

feedback. Data sets $\left(E_{i 1}, F_{i 1}, G_{i 1}\right)$ and $\left(E_{i 2}, F_{i 2}, G_{i 2}\right)$ correspond to $t=n T$ and $t=(2 n-1) T, n=1,2,3, \cdots$, respectively, where $i=1$ and $i=2$ correspond to the position $x$ and velocity $\dot{x}$ of the state vector $\mathbf{x}$ 
the most by the LQR controller, the delayed position also has a contribution with more weight assigned to the other end of the delay interval.

\section{Optimal control via non-delayed state feedback using CSCTA, RLFT, and time-invariant LQR}

The third strategy for obtaining a non-delayed control law is adopted from $[18,19]$ and involves use of both CSCTA and RLFT as well as pole-placement for LTI systems. First, Eq. (8) which is in the form of Eq. (1) is discretized using CSCTA and is written in the form of Eq. (5). The LFT [17, $18,22,31]$

$\mathbf{y}(t)=\mathbf{Q}(t) \mathbf{z}(t)$,

where the square matrix $\mathbf{Q}(t)$ is a real $2 T$-periodic matrix that has the same size as $\hat{\mathbf{A}}$ in Eq. (6), is used to transform the uncontrolled ODE in Eq. (5) to

$\dot{\mathbf{z}}=\mathbf{R z}(t)$.

Furthermore, the eigenvalues of $\mathbf{R}$ include the dominant characteristic exponents of the uncontrolled periodic DDE (1). It has been shown in [31] that the LFT matrix $\mathbf{Q}(t)$ can be computed in closed form for a commutative system for which the state transition matrix (STM) is known. In general, the STM of the uncontrolled system in Eq. (5) can be factored as

$\boldsymbol{\Phi}(t)=\mathbf{Q}(t) e^{\mathbf{R} t}$

where the real matrix $\mathbf{R}$ is obtained as

$\mathbf{R}=\frac{1}{2 T} \ln \boldsymbol{\Phi}(2 T)$.

However, for the DDE system a RLFT is required in which only the $r$ modes corresponding to $r$ dominant Floquet multipliers are retained, since the inaccurate multipliers clustered around the origin result in a set of inaccurate eigenvalues of $\mathbf{R}$ (characteristic exponents) with highly negative real parts which in turn makes it impractical to compute the full LFT matrix $\mathbf{Q}(t)$. Often we choose $r=q$ for the stable parameter set, that is, we retain the same number of modes as the size of the DDE. For the unstable parameter set, however, the dominant modes are those with magnitudes more than 1 as well as those with magnitudes very close to 1 . Therefore, consider an order reduction transformation

$\mathbf{z}=\hat{\mathbf{T}} \mathbf{w}$

where

$$
\begin{aligned}
\hat{\mathbf{T}} & =\mathbf{M T M}_{11}^{-1}=\left[\begin{array}{ll}
\mathbf{M}_{11} & \mathbf{M}_{12} \\
\mathbf{M}_{21} & \mathbf{M}_{22}
\end{array}\right]\left[\begin{array}{c}
\mathbf{I}_{q} \\
\mathbf{0}_{(m-1) q \times q}
\end{array}\right] \mathbf{M}_{11}^{-1} \\
& =\left[\begin{array}{c}
\mathbf{I}_{q} \\
\mathbf{M}_{21} \mathbf{M}_{11}^{-1}
\end{array}\right]
\end{aligned}
$$

is a $m q \times q$ rectangular matrix, $\mathbf{M}$ is the modal matrix of $\mathbf{R}$ such that $\mathbf{R}=\mathbf{M} \mathbf{\Lambda} \mathbf{M}^{-1}$, and the matrix

$\boldsymbol{\Lambda}=\left[\begin{array}{cc}\boldsymbol{\Lambda}_{1} & \mathbf{0} \\ \mathbf{0} & \boldsymbol{\Lambda}_{2}\end{array}\right]$

is diagonal. Only the modal coordinates corresponding to $\boldsymbol{\Lambda}_{1}$ are retained. Also, $\mathbf{M}_{11}$ is the top left $q \times q$ partition of $\mathbf{M}$. Application to Eq. (24) yields [32]

$\dot{\mathbf{w}}=\overline{\mathbf{R}} \mathbf{w}$

where $\overline{\mathbf{R}}=\left(\hat{\mathbf{T}}^{T} \hat{\mathbf{T}}\right)^{-1} \hat{\mathbf{T}}^{T} \mathbf{R} \hat{\mathbf{T}}$ is a projection of matrix $\mathbf{R}$ onto w such that Eq. (30) exactly preserves the projected eigenstructure of Eq. (24) including the dominant eigenvalues and corresponding projected modes. Note that in Eq. (27) $\mathbf{z}_{1}=\mathbf{w}$ (master coordinates) and $\mathbf{z}_{2}=\mathbf{M}_{21} \mathbf{M}_{11}^{-1} \mathbf{w}$ is the relation between master and slave coordinates where $\mathbf{z}=\left[\begin{array}{ll}\mathbf{z}_{1}^{T} & \mathbf{z}_{2}^{T}\end{array}\right]^{T}$ is partitioned into vectors of length $q$ and $(m-1) q$, respectively. For $m=3$ Chebyshev collocation points, for instance, the matrices described above are obtained for the delayed Mathieu equation given in Eq. (8) as

$\mathbf{R}=\left[\begin{array}{cccccc}0 & 1.0107 & -0.0005 & 0 & 0.0005 & 0 \\ -0.1998 & 0 & -0.0007 & 0 & 0.5013 & 0 \\ 1.0012 & 0 & 0.0007 & 0 & -1.0048 & 0 \\ -0.0010 & 1.0126 & -0.0015 & 0 & 0.0011 & -1.0050 \\ -1.0040 & -0.0019 & 4.0211 & 0 & -3.0156 & 0 \\ -0.0181 & -1.0047 & -0.0001 & 4.0200 & 0.0018 & -3.0150\end{array}\right] \in \mathbb{R}^{6 \times 6}$

$\mathbf{M}=\left[\begin{array}{cccc}0.6375 & 0.2206 & 0.0540 \mp 0.0451 i & 0 \\ 0.2395 & -0.2287 & -0.0045 \pm 0.1220 i & 0 \\ 0.5276 & 0.3321 & -0.0147 \mp 0.2480 i & 0 \\ 0.2013 & -0.3455 & 0.3388 \pm 0.2706 i & 0.3354 \pm 0.2958 i \\ 0.4362 & 0.5661 & -0.2848 \mp 0.3157 i & 0 \\ 0.1643 & -0.5906 & 0.7414 & 0.8944\end{array}\right] \in \mathbb{R}^{6 \times 6}$,

$\mathbf{\Lambda}=\operatorname{diag}([0.3796,-1.0472,-1.1737 \pm 1.3021 i$,

$-1.5075 \pm 1.3295 i] \in \mathbb{R}^{6 \times 6}$,

$\hat{\mathbf{T}}=\left[\begin{array}{cc}1 & 0 \\ 0 & 1 \\ 1.0079 & -0.4798 \\ -0.1849 & 1.3325 \\ 1.1849 & -1.3325 \\ -0.5230 & 2.0780\end{array}\right] \in \mathbb{R}^{6 \times 2}, \quad \overline{\mathbf{R}}=\left[\begin{array}{cc}0.0001 & 1.0103 \\ 0.3934 & -0.6677\end{array}\right] \in \mathbb{R}^{2 \times 2}$

where the eigenvalues of $\overline{\mathbf{R}}(0.3796,-1.0472)$ are the dominant eigenvalues (characteristic exponents) of $\mathbf{R}$.

Because the state vector $\mathbf{x}(t)$ is actually a reduced subset of the first $q$ elements of vector $\mathbf{y}(t)$, we define a $q \times q$ RLFT matrix $\overline{\mathbf{Q}}(t)$ that transforms the uncontrolled DDE in Eq. (1) into Eq. (30) and that satisfies

$$
\begin{aligned}
\mathbf{x}(t) & =\mathbf{T}^{T} \mathbf{y}(t)=\mathbf{T}^{T} \mathbf{Q}(t) \mathbf{z}(t)=\overline{\mathbf{Q}}(t) \mathbf{w}(t) \\
& =\overline{\mathbf{Q}}(t)\left(\hat{\mathbf{T}}^{T} \hat{\mathbf{T}}\right)^{-1} \hat{\mathbf{T}}^{T} \mathbf{z}(t)
\end{aligned}
$$


where $\mathbf{T}=\left[\begin{array}{ll}\mathbf{I}_{q} & \mathbf{0}_{q \times(m-1) q}\end{array}\right]^{T}$ and

$\overline{\mathbf{Q}}(t)=\mathbf{T}^{T} \mathbf{Q}(t) \hat{\mathbf{T}}$.

Equation (32) represents a projection of the full LFT matrix. Note, however, that since it is impractical to compute $\mathbf{Q}(t)$ in its entirety to begin with, an alternative formula which was used successfully for an approximate $\mathbf{\mathbf { Q }}(t)$ in [22] is implemented to obtain the $2 T$-periodic $\overline{\mathbf{Q}}(t)$ as

$\overline{\mathbf{Q}}(t)=\boldsymbol{\Phi}_{11}(t) e^{-\mathbf{R}_{11} t}$.

where $\boldsymbol{\Phi}_{11}(t)$ is the upper $q \times q$ partition of the Jordan form of the modes of $\boldsymbol{\Phi}(t)$ sorted in a manner that dominant modes are the top diagonal elements, and $\mathbf{R}_{11}$ is the upper $q \times q$ partition of the $\mathbf{R}$ matrix where $q=2$ is the order of the system given in Eq. (8). The $l$-term Fourier expansion of the elements of the RLFT matrix can be expressed as

$\bar{Q}_{i j}(t)=\frac{a_{0}^{i j}}{2}+\sum_{n=1}^{l} a_{n}^{i j} \cos \left(\frac{n \Omega t}{2}\right)+\sum_{n=1}^{l} b_{n}^{i j} \sin \left(\frac{n \Omega t}{2}\right)$.

For $m=3$ Chebyshev collocation points, the 5-term Forier expansion of the elements of matrix $\overline{\mathbf{Q}}(t)$ are obtained for the delayed Mathieu equation given in Eq. (8) as

$$
\begin{aligned}
\bar{Q}_{11}(t)= & 0.999139-0.001363 \sin \pi t+0.001930 \cos \pi t \\
& +0.001874 \cos 2 \pi t-0.003555 \sin 2 \pi t \\
& -0.001000 \cos 3 \pi t-0.000271 \sin 3 \pi t \\
& -0.000474 \cos 4 \pi t-0.000075 \sin 4 \pi t \\
& -0.000288 \cos 5 \pi t-0.000028 \sin 5 \pi t \\
\bar{Q}_{12}(t)= & 0.001552-0.003304 \cos \pi t+0.006344 \sin \pi t \\
& +0.000314 \cos 2 \pi t+0.005429 \sin 2 \pi t \\
& +0.001869 \cos 3 \pi t+0.002464 \sin 3 \pi t \\
& +0.000974 \cos 4 \pi t+0.001270 \sin 4 \pi t \\
& +0.000617 \cos 5 \pi t+0.000895 \sin 5 \pi t \\
\bar{Q}_{21}(t)= & 0.003117+0.000033 \cos \pi t+0.000972 \sin \pi t \\
& -0.001980 \cos 2 \pi t-0.018526 \sin 2 \pi t \\
& -0.000038 \cos 3 \pi t+0.000033 \sin 3 \pi t \\
& -0.000023 \cos 4 \pi t+0.000176 \sin 4 \pi t \\
& -0.000000 \cos 5 \pi t+0.000191 \sin 5 \pi t \\
& +998171-0.002036 \cos \pi t-0.001120 \sin \pi t \\
& -0.001973 \cos 2 \pi t+0.000696 \sin 2 \pi t \\
& +0.001018 \cos 3 \pi t-0.00054 \sin 3 \pi t \\
& +0.000468 \cos 4 \pi t-0.000564 \sin 4 \pi t \\
& +0.000259 \cos 5 \pi t-0.000495 \sin 5 \pi t \\
\bar{Q}_{22}(t)= & 0.95 \\
& \\
& 0.04
\end{aligned}
$$

According to [32], application of RLFT with $q$ dominant modes to Eq. (5) yields

$\dot{\mathbf{w}}(t)=\overline{\mathbf{R}} \mathbf{w}(t)+\left(\hat{\mathbf{T}}^{T} \hat{\mathbf{T}}\right)^{-1} \hat{\mathbf{T}}^{T} \mathbf{Q}^{-1}(t) \hat{\mathbf{B}}(t) \mathbf{u}(t)$,

It is desired to express Eq. (36) in terms of the projection $\overline{\mathbf{Q}}(t)$ instead of the full LFT matrix $\mathbf{Q}(t)$. Taking the transpose of both sides of Eq. (32) yields

$\overline{\mathbf{Q}}^{T}(t)=\hat{\mathbf{T}}^{T} \mathbf{Q}^{T}(t) \mathbf{T}$

The minimum norm inverse for $\mathbf{Q}^{T}(t) \mathbf{T}$ can be obtained from Eq. (37) as

$\mathbf{Q}^{T}(t) \mathbf{T}=\hat{\mathbf{T}}\left(\hat{\mathbf{T}}^{T} \hat{\mathbf{T}}\right)^{-1} \overline{\mathbf{Q}}^{T}(t)$.

Taking the transpose of both sides of Eq. (38) yields

$\overline{\mathbf{Q}}(t)\left(\hat{\mathbf{T}}^{T} \hat{\mathbf{T}}\right)^{-1} \hat{\mathbf{T}}^{T}=\mathbf{T}^{T} \mathbf{Q}(t)$.

If we premultiply and postmultiply both sides of Eq. (39) with $\overline{\mathbf{Q}}^{-1}(t)$ and $\mathbf{Q}^{-1}(t)$, respectively, we obtain

$\left(\hat{\mathbf{T}}^{T} \hat{\mathbf{T}}\right)^{-1} \hat{\mathbf{T}}^{T} \mathbf{Q}^{-1}(t)=\overline{\mathbf{Q}}^{-1}(t) \mathbf{T}^{T}$.

Substituting Eq. (40) into Eq. (36), the latter can be written as

$\dot{\mathbf{w}}(t)=\overline{\mathbf{R}} \mathbf{w}(t)+\overline{\mathbf{Q}}^{-1}(t) \mathbf{B}(t) \mathbf{u}(t)$

with the initial conditions expressed as

$\mathbf{w}_{0}=\mathbf{M}_{11} \mathbf{T}^{T} \mathbf{M}^{-1} \mathbf{y}_{0}$

where $\mathbf{y}_{0}=\left[\begin{array}{llll}1 & 0 & \cdots & 0\end{array}\right]^{T}$ is the initial condition for the discretized system obtained using CSCTA.

Following [18], we consider the auxiliary system

$\dot{\overline{\mathbf{w}}}(t)=\overline{\mathbf{R}} \overline{\mathbf{w}}(t)+\overline{\mathbf{B}} \overline{\mathbf{v}}(t)$

with the initial conditions expressed as [22], where $\overline{\mathbf{B}}$ is an arbitrary constant matrix with the same order and rank as $\overline{\mathbf{Q}}^{-1}(t) \mathbf{B}(t)$ such that $(\overline{\mathbf{R}}, \overline{\mathbf{B}})$ is a controllable pair and $\overline{\mathbf{v}}(t)$ is the control force. Then, we design a feedback control as

$\overline{\mathbf{v}}(t)=-\overline{\mathbf{K}} \overline{\mathbf{w}}(t)$

such that $(\overline{\mathbf{R}}-\overline{\mathbf{B}} \overline{\mathbf{K}})$ is Hurwitz. The control gain $\overline{\mathbf{K}}$ can be designed by applying pole-placement or optimal control using time-invariant LQR. Defining $\mathbf{e}(t)=\mathbf{w}(t)-\overline{\mathbf{w}}(t)$, the error dynamics from Eqs. (41) and (43) are given as

$$
\begin{aligned}
\dot{\mathbf{e}}(t)= & \dot{\mathbf{w}}(t)-\dot{\overline{\mathbf{w}}}(t)=(\overline{\mathbf{R}}-\overline{\mathbf{B}} \overline{\mathbf{K}}) \mathbf{e}(t)+\overline{\mathbf{B}} \overline{\mathbf{K}} \mathbf{w}(t) \\
& +\overline{\mathbf{Q}}^{-1}(t) \mathbf{B}(t) \mathbf{u}(t) .
\end{aligned}
$$

To ensure that the error goes to zero, the sum of the other two rightmost terms should vanish, i.e.

$\overline{\mathbf{Q}}^{-1}(t) \mathbf{B}(t) \mathbf{u}(t)=-\overline{\mathbf{B}} \overline{\mathbf{K}} \mathbf{w}(t)$. 
Premultiplying both sides by $\overline{\mathbf{Q}}(t)$ and using the least squares inverse to solve for $\mathbf{u}(t)$, the control law is obtained as

$\mathbf{u}(t)=-\left(\mathbf{B}^{T}(t) \mathbf{B}(t)\right)^{-1} \mathbf{B}^{T}(t) \overline{\mathbf{Q}}(t) \overline{\mathbf{B}} \overline{\mathbf{K}} \mathbf{w}(t)$

in terms of the coordinates $\mathbf{w}(t)$. Considering Eqs. (23) and (27), $\mathbf{w}(t)$ can be expressed in terms of $\mathbf{y}(t)$ as

$\mathbf{w}(t)=\left(\hat{\mathbf{T}}^{T} \hat{\mathbf{T}}\right)^{-1} \hat{\mathbf{T}}^{T} \mathbf{Q}^{-1}(t) \mathbf{y}(t)$.

Substituting Eq. (48) into Eq. (47), using Eq. (40) to express $\mathbf{Q}^{-1}(t)$ in terms of $\overline{\mathbf{Q}}^{-1}(t)$, and using Eq. (31) to express $\mathbf{y}$ in terms of $\mathbf{x}$, the control law can be expressed as

$\mathbf{u}(t)=-\mathbf{K}(t) \mathbf{x}(t)$,

where the periodic gain matrix is

$\mathbf{K}(t)=\left(\mathbf{B}^{T}(t) \mathbf{B}(t)\right)^{-1} \mathbf{B}^{T}(t) \overline{\mathbf{Q}}(t) \overline{\mathbf{B}} \overline{\mathbf{K}} \overline{\mathbf{Q}}^{-1}(t)$.
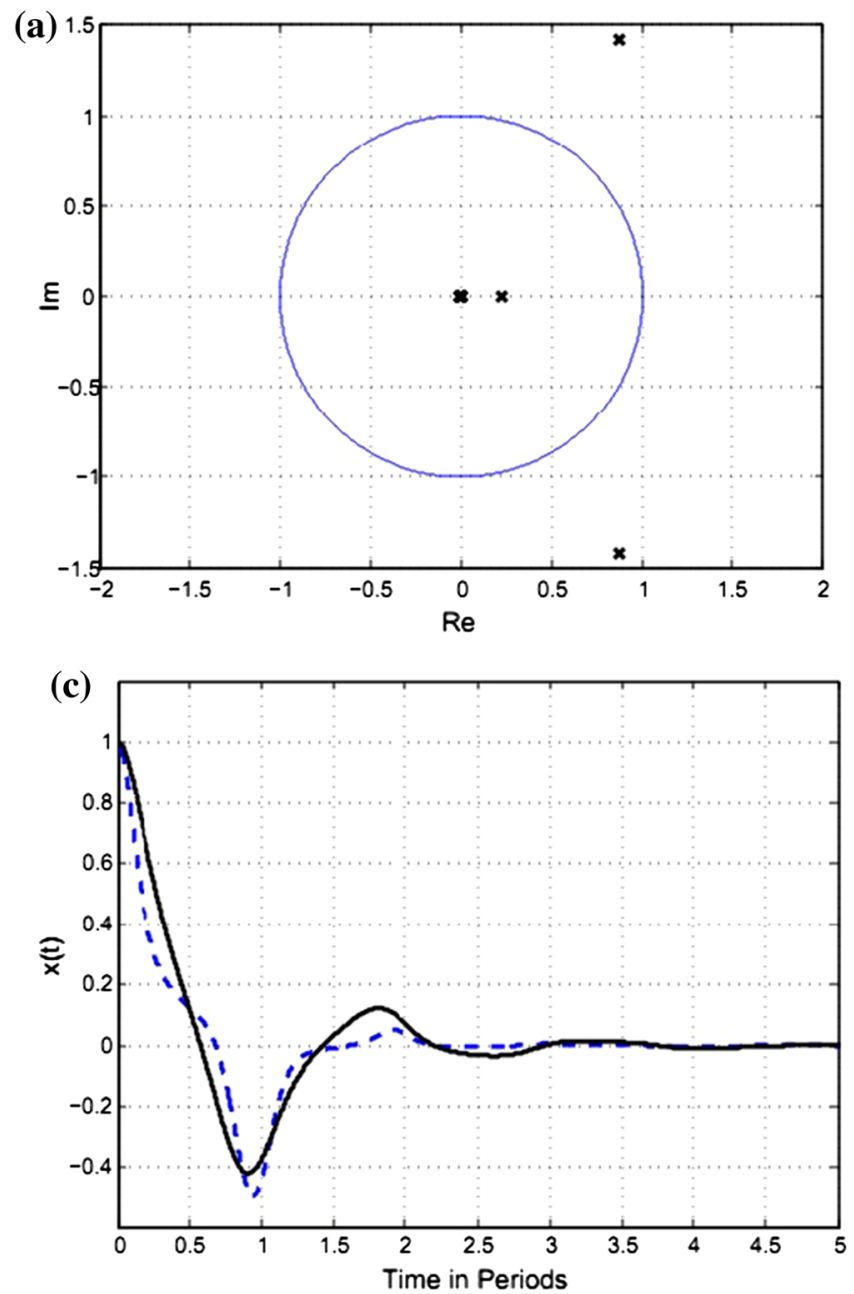

Fig. 12 a Squares of the Floquet multipliers, $\mathbf{b}$ control gains obtained by RLFT and time-invariant LQR method (with the weighting matrices $\mathbf{R}=1$ and $\mathbf{Q}=\mathbf{I}_{q \times q}$ ), c controlled response, and $\mathbf{d}$ control force using the control law given by Eqs. (49-50) for the delayed Mathieu equation
This is an optimal control via current state feedback since time-invariant LQR is used for the auxiliary LTI system given in Eq. (43).

As is discussed in [33], due to use of the least squares inverse, asymptotic stability of the closed-loop dynamics is not guaranteed and one must search for a $\overline{\mathbf{B}}$ matrix that provides an acceptable response. Alternatively, the use of a backstepping strategy as detailed in [33] guarantees asymptotic stability for underactuated systems, although that is not employed here. In practice, $\overline{\mathbf{Q}}^{-1}(t)$ can be evaluated in two different ways: (1) inverting the $\overline{\mathbf{Q}}(t)$ given in Eq. (33) at each time step and (2) finding the STM $\Psi(t)$ associated with the adjoint system (as is explained in [19,20,33-35]) as

$\dot{\mathbf{s}}(t)=-\hat{\mathbf{A}}^{T}(t) \mathbf{S}(t)$

and using the relationships $\boldsymbol{\Phi}^{-1}(t)=\boldsymbol{\Psi}^{T}(t)$ which yields
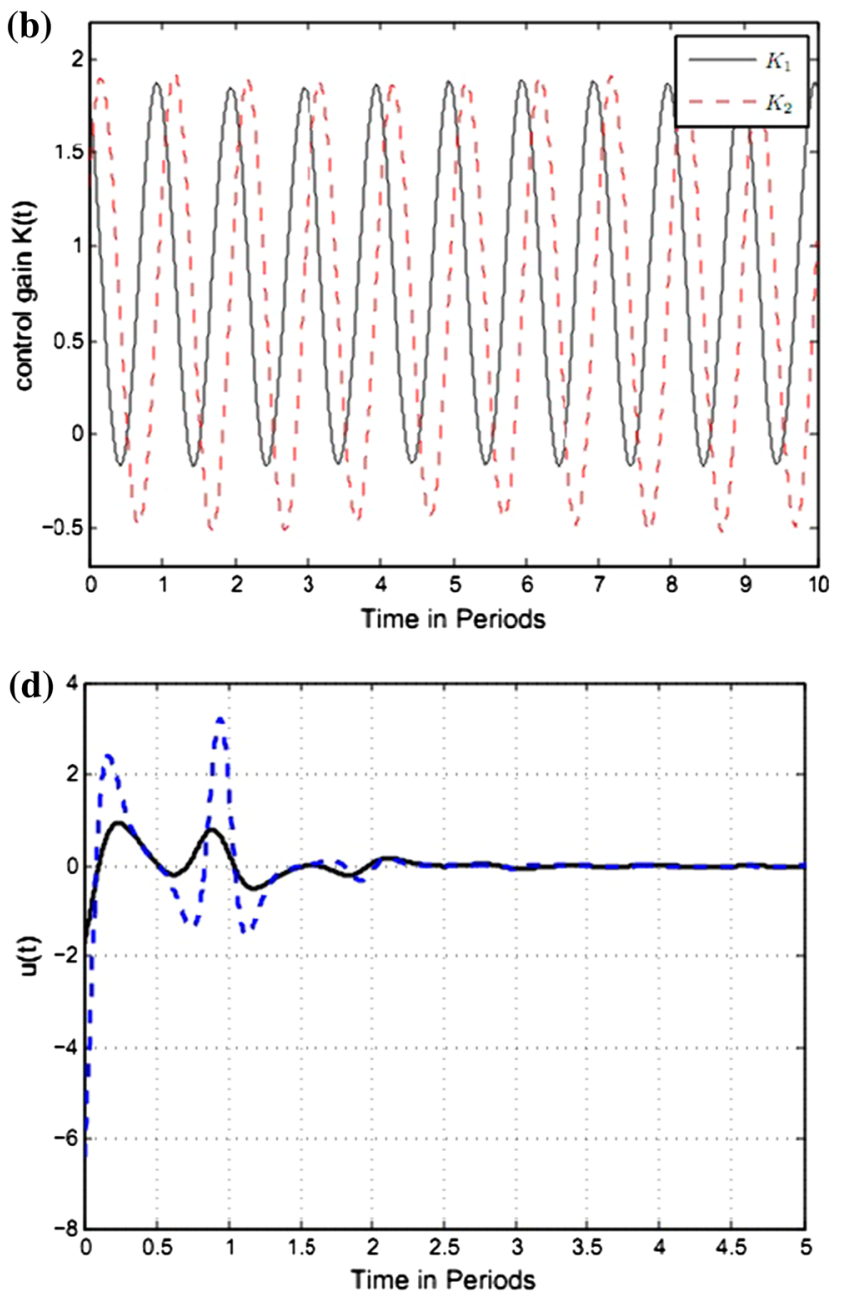

given by Eq. (8) with $a=1, b=0.1, c=0.5, \Omega=2$, and $\tau=\pi$. In parts $\mathbf{c}, \mathbf{d}$, the results obtained by RLFT and pole-placement with poles $[-0.4-0.3]($ dash) are compared with those obtained by RLFT and time-invariant LQR (solid). In the simulations, $\overline{\mathbf{B}}=\mathbf{B}$ 
$\overline{\mathbf{Q}}^{-1}(t)=e^{\mathbf{R}_{11} t} \boldsymbol{\Psi}_{11}^{T}(t)$

The implementation of the control law defined by Eqs. (4950) is now demonstrated for the delayed Mathieu equation given in Eq. (8) with two sets of parameter values. First, the parameter values are chosen to be $a=1, b=0.1, c=$ $0.5, \Omega=2$, and $\tau=\pi$ for $\mathbf{B}=[1,0]^{T}$ and $m=20$ Chebyshev collocation points are used in the CSCTA. This parameter set corresponds to parametric resonance and hence instability in the uncontrolled system. The squares of the Floquet multipliers, the elements of the fundamental matrix $\boldsymbol{\Phi}(t)$ corresponding to the dominant modes, elements of the RLFT matrix $\overline{\mathbf{Q}}(\mathbf{t})$, uncontrolled response, control gains obtained by time-invariant $\mathrm{LQR}$ method using the control law given by Eqs. (49-50), and the results obtained by the implementation of that control law are shown in Fig. 12. The total con-

(a)

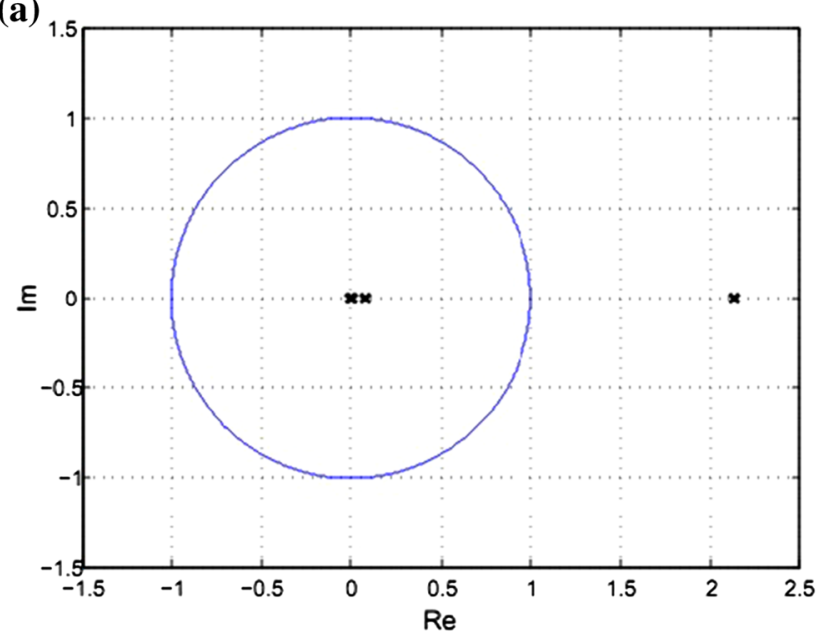

(c)

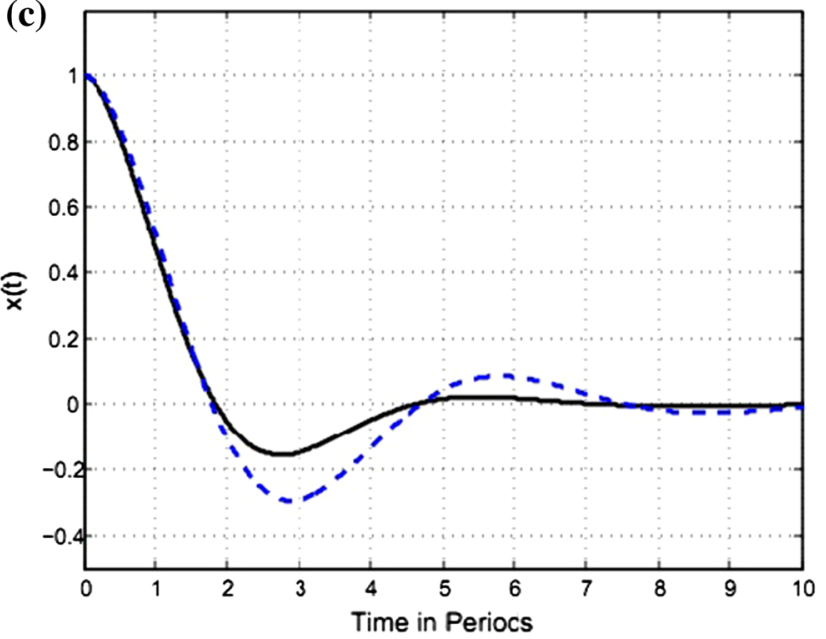

Fig. 13 a Squares of the Floquet multipliers, $\mathbf{b}$ control gains obtained by RLFT and time-invariant LQR method (with the weighting matrices $\mathbf{R}=1$ and $\mathbf{Q}=\mathbf{I}_{q \times q}$ ), $\mathbf{c}$ controlled response, and $\mathbf{d}$ control force using the control law given by Eqs. (49-50) for the delayed Mathieu equation trol efforts for the time-invariant LQR and pole-placement approaches are obtained as $\int_{0}^{\infty}|u(\eta)| d \eta=3.9531$ and $\int_{0}^{\infty}|u(\eta)| d \eta=3.9734$, respectively, when the control law defined by Eqs. (49-50) is used. Next, the parameter values are chosen to be the same as those studied in Sects. 3 and 4, i.e. $a=0.2, b=0.1, c=0.5, \Omega=2 \pi$, and $\tau=1$. The squares of the Floquet multipliers of the uncontrolled system, the control gains $\mathbf{K}(t)$ and control effort obtained by the time-invariant $L Q R$ method to solve for the $\overline{\mathbf{K}}$ matrix in Eq. (44) (with $\overline{\mathbf{B}}=\mathbf{B}$ and the weighting matrices $\mathbf{R}=1$ and $\mathbf{Q}=\mathbf{I}_{q \times q}$ ) and via pole-placement (with poles $[-0.7-1]$ ) are shown in Fig. 13. The total control effort is obtained as $\int_{0}^{t_{f}}|u(\eta)| d \eta=1.80$ and 1.55 when the pole-placement and time-invariant LQR approaches are used, respectively.

Therefore, it is seen that the combined use of CSCTA and RLFT allows for the linear uncontrolled periodic DDE to be
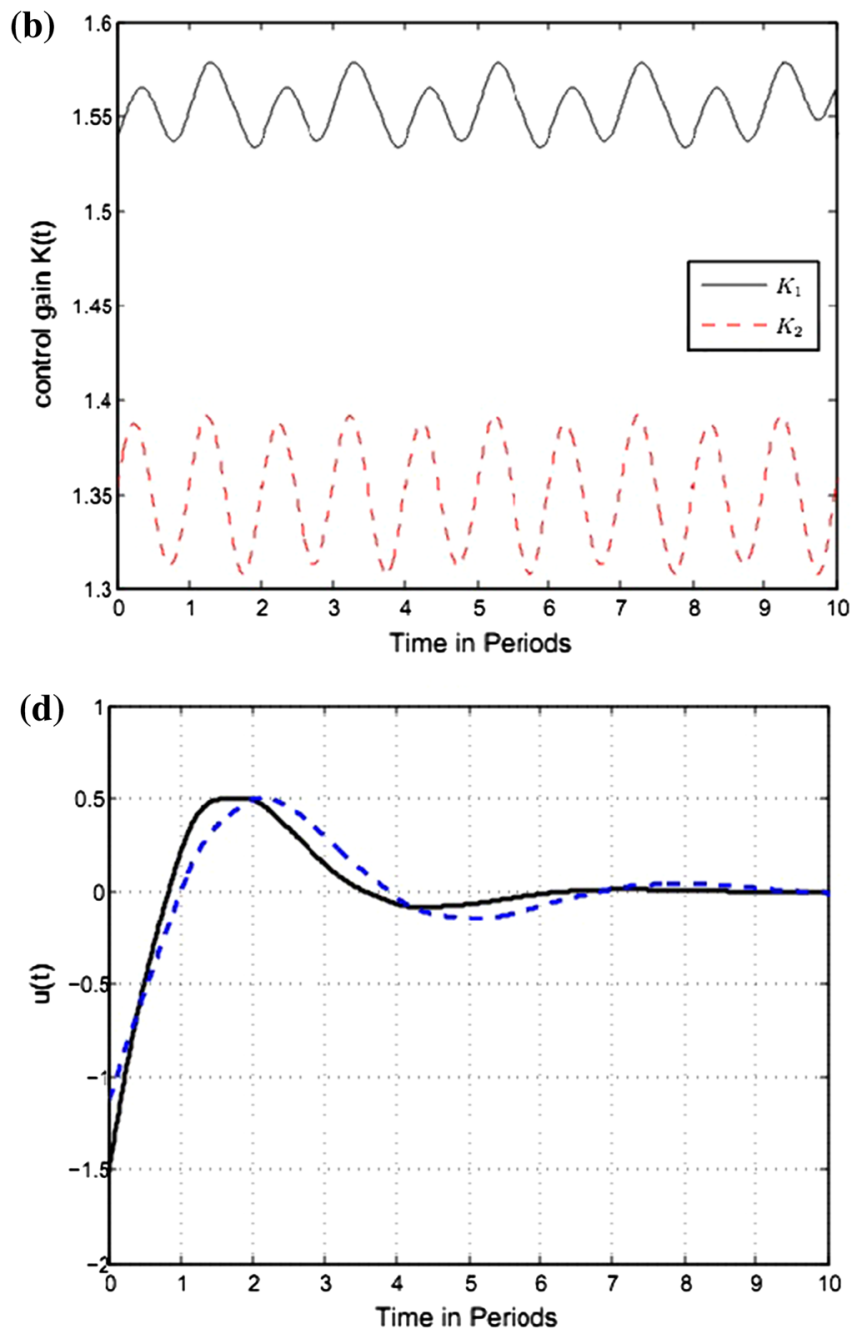

given by Eq. (8) with $a=0.2, b=0.1, c=0.5, \Omega=2 \pi$, and $\tau=1$. In parts $\mathbf{c}, \mathbf{d}$, the results obtained by RLFT and pole-placement with poles $[-0.7-1]($ dash) are compared with those obtained by RLFT and time-invariant LQR (solid). In the simulations, $\overline{\mathbf{B}}=\mathbf{B}$ 
Table 1 Total control effort and settling time of the closed-loop response for the three proposed optimal control strategies

\begin{tabular}{|c|c|c|c|}
\hline & Total control eff & fort & Settling time in periods \\
\hline 1 st control strategy & 1.7792 & & 14 \\
\hline 2nd control strategy & $\begin{array}{l}\text { PRE } \\
\text { periodic ARE }\end{array}$ & $\begin{array}{l}0.810 \\
0.891\end{array}$ & 6 \\
\hline 3rd control strategy & 1.55 & & 7 \\
\hline
\end{tabular}

expressed in an equivalent time-invariant and non-delayed form which preserves the dominant characteristic exponents. Although this strategy is implemented for a linear periodic time-delayed system, the same procedure can be for nonlinear systems, within a region of attraction, where the linear part can be transformed. Consequently, many additional tools for analysis and control of nonlinear time-varying systems can be used. Note, again, that the combined use of CSCTA and RLFT has thus far not been investigated for their application in optimal feedback control design for delayed periodic systems.

\section{Comparison and conclusions}

Three optimal feedback control strategies using periodic control gains were proposed for linear periodic time delayed systems. The first strategy, which can be considered as a linear delayed feedback control with discrete delay, used infinitedimensional Floquet theory to obtain the stability domains in the parameter space of control gains, where Chebyshev collocation method was used to approximate the monodromy operator. The optimal set of control gains was obtained based on the minimum spectral radius. The second strategy, which can be considered as a linear delayed feedback control with distributed delay, used time-varying LQR control to minimize a cost function by directly solving the time-PRE backwards in time (or alternatively the corresponding periodic ARE), in which the time-periodic DDE was transformed into a large set of ODEs by using CSCTA technique. The optimal control gain was obtained for different weighting matrices. The third strategy, which can be considered as nondelayed feedback control, used both CSCTA and RLFT as well as either pole-placement or time-invariant LQR to obtain the control gains that asymptotically stabilize the auxiliary reduced order LTI system, and hence the original system as well.

These strategies were introduced into the delayed Mathieu equation and the closed-loop response of the controlled system, control force, and the total control effort were studied for each control strategy. While the first strategy is the easiest to implement to the system, it requires more control effort for the same settling time compared to the other two control strategies. On the other hand, not only does the second control strategy require the least control effort, but also the closed-loop response of the system using the second con- trol strategy has the least settling time. However, in the third control strategy, only the dominant modes of the monodromy matrix were employed to design the control law. Therefore, the third control strategy is computationally more efficient, compared to the other two, while its settling time remains reasonably small. However, due to use of the least squares inverse in the RLFT technique, asymptotic stability of the closed-loop response is not guaranteed and searching for an arbitrary $\overline{\mathbf{B}}$ matrix as well as the weighting state and input matrices for the time-invariant LQR that provide an acceptable response can be challenging. The total control effort and settling time of the three control strategies are compared in Table 1 where only the current states are weighted in the cost function for the second control strategy.

As a future work, the backstepping technique can be added to RLFT to guarantee asymptotic stability of the closed-loop response when the third control strategy is used. Another possibility could be the use of Chebyshev collocation technique to obtain a discretized map and the use of constant-gain feedback control such as time-invariant LQR for the resulting discretized map. In addition, the act-and-wait control strategy [2,8-10] could possibly be combined with some of the ideas for optimal feedback control of time-delayed periodic systems presented here.

Acknowledgments Financial support from the National Science Foundation under Grant No. CMMI-1131646 is gratefully acknowledged.

\section{References}

1. Niculescu S-I (2001) Delay effects on stability: a robust control approach. Springer, London

2. Insperger T, Stépán G (2011) Semi-discretization for time-delay systems: stability and engineering applications. Springer, New York

3. Ma H, Deshmukh V, Butcher E, Averina V (2005) Delayed state feedback and chaos control for time-periodic systems via a symbolic approach. Commun Nonlinear Sci Numer Simul 10:479-497

4. Manitius A, Olbrot A (1979) Finite spectrum assignment problem for systems with delays. IEEE Trans Autom Control 24:541-553

5. Engelborghs K, Dambrine M, Roose D (2001) Limitations of a class of stabilization methods for delay systems. IEEE Trans Autom Control 46:336-339

6. Mondie S, Dambrine M, Santos O (2002) Approximation of control laws with distributed delays: a necessary condition for stability. Kybernetika 38:541-551

7. Mondie S, Michiels W (2003) Finite spectrum assignment of unstable time-delay systems with a safe implementation. IEEE Trans Autom Control 48:2207-2212 
8. Insperger T (2006) Act and wait concept for time-continuous control systems with feedback delay. IEEE Trans Control Syst 14:974977

9. Stepan G, Insperger T (2006) Stability of time-periodic and delayed systems: a route to act and wait control. Ann Rev Control 30:159168

10. Insperger T, Stepan G (2007) Act and wait concept for discrete-time systems with feedback delay. IET Control Theory A 1:553-557

11. Sun J-Q, Song B (2009) Control studies of time-delayed dynamical systems with the method of continuous time approximation. Commun Nonlinear Syst Numer Simul 14(11):3933-3944

12. Wahi P, Chatterjee A (2005) Galerkin projections for delay differential equations. J Dyn Syst Meas Control 127:80-87

13. Butcher E, Bobrenkov O (2011) On the chebyshev spectral continuous time approximation for constant and periodic delay differential equations. Commun Nonlinear Sci Numer Simul 16:1541-1554

14. Butcher E, Bobrenkov O, Bueler E, Nindujarla P (2009) Analysis of milling stability by the chebyshev collocation method: algorithm and optimal stable immersion levels. J Comput Nonlinear Dyn 3:112

15. Elbeyli O, Sun J-Q (2004) On the semi-discretization method for feedback control design of linear systems with time delay. J Sound Vib 273:429-440

16. Butcher E, Mann B (2009) Stability analysis and control of linear periodic delayed systems using chebyshev and temporal finite element methods. In: Balachandran B, Gilsinn D, Kalmar-Nagy T (eds) Delay differential equations, recent advances and new directions. Springer, New York

17. Sinha S, Pandiyan R, Bibb J (1996) Liapunov-Floquet transformation: computation and applications to periodic systems. J Vib Acoust 118:209-219

18. Sinha S, Joseph P (1994) Control of general dynamic systems with periodically varying parameters via Liapunov-Floquet transformation. J Dyn Syst Meas Control 116:650-658

19. Deshmukh V, Sinha S, Joseph P (2000) Order reduction and control of parametrically excited dynamical systems. J Vib Control 6:1017-1028

20. Dávid A, Sinha S (2003) Bifurcation control of nonlinear systems with time-periodic coefficients. J Dyn Syst Meas Control 125:541548

21. Sinha S, David A (2006) Control of chaos in nonlinear systems with time-periodic coefficients. Philos Trans R Soc A 364:2417-2432

22. Bobrenkov O, Butcher E, Mann B (2012) On application of the Liapunov-Floquet transformation to differential equations with time delay and periodic coefficients. J Vib Control 2:1-8. doi:10. $1177 / 1077546311433914$
23. Bobrenkov OA (2011) Analysis of periodic systems with time delay via chebyshev spectral collocation with application to milling. $\mathrm{PhD}$ thesis, New Mexico State University (August, 2011)

24. Trefethen L (2000) Spectral methods in MATLAB. SIAM Press, Philadelphia

25. Bueler E (2007) Error bounds for approximate eigenvalues of periodic-coefficient linear delay differential equations. SIAM J Numer Anal 45(6):2510-2536

26. Bobrenkov O, Nazari M, Butcher E (2012) Response and stability analysis of periodic delayed systems with discontinuous distributed delay. J Comput Nonlinear Dyn 7(3):4

27. Papoulis A (1967) Limits on bandlimited signals. Proc IEEE 55(10):1677-1686

28. Hassouneh M, Lee H-C, Abed E (2004) Washout filters in feedback control: benefits, limitations and extensions. Proceedings of the American Control Conference, Boston, pp 3950-3955

29. Bittanti S, Colaneri P, Nicolao G (1991) The periodic riccati equation. In: Bittanti S, Laub A, Willems J (eds) The riccati equation. Springer, New York, pp 127-162

30. Friedland B (1987) Control system design: an introduction to statespace methods. McGraw-Hill, New York

31. Sinha S, Pandiyan R (1994) Analysis of quazilinear systems with periodic coefficients via Liapunov-Floquet transformation. Int J Non-Linear Mech 29(5):687-702

32. Butcher E, Bobrenkov O, Nazari M, Torkamani S (2012) Estimation and control in time-delayed dynamical systems using the chebyshev spectral continuous time approximation and reduced Liapunov-Floquet transformation. In: Sun J-Q, Ding Q (eds) Recent advances in analysis and control of time-delayed dynamical systems. SIAM, Philadelphia

33. Deshmukh V, Sinha S (2004) Control of dynamic systems with time-periodic coefficients via the Lyapunov-Floquet transformation and backstepping technique. J Vib Control 10:1517-1533

34. David A, Sinha S (1999) Some ideas on the local control of nonlinear systems with time-periodic coefficients. Proceedings of the ASME design engineering technical conferences, Las Vegas, Sept 12-15, 1999, pp 1-10

35. Pandiyan R, Sinha S (2001) Time-varying controller synthesis for nonlinear systems subjected to periodic parametric loadings. J Vib Control 7:73-90 